\title{
Discovery of an MIT-like atracotoxin family: spider venom peptides that share sequence homology but not pharmacological properties with AVIT family proteins
}

\author{
Suping Wena, David T. R. Wilson', Sanjaya Kuruppu', Michael L. J. Korsinczky ${ }^{\mathrm{b}}$, \\ Joseph Hedrick ${ }^{d}$, Ling Pang ${ }^{d}$, Tim Szeto ${ }^{\mathrm{e}}{ }^{\dagger}$, Wayne C. Hodgson ${ }^{\mathrm{c}}$, Paul F. Alewood ${ }^{\mathrm{b}}$, \\ Graham M. Nicholson ${ }^{\text {a,* }}$ \\ ${ }^{a}$ Neurotoxin Research Group, Department of Health Sciences, University of Technology, Sydney, PO \\ Box 123, Broadway NSW 2007, Australia \\ ${ }^{b}$ Institute for Molecular Biosciences, University of Queensland, St. Lucia, QLD 4072, Australia \\ ${ }^{c}$ Monash Venom Group, Department of Pharmacology, Monash University, Clayton, Victoria 3800, \\ Australia \\ ${ }^{d}$ Schering-Plough Corporation, 2000 Galloping Hill Rd, Kenilworth NJ 07033-1329, USA \\ ${ }^{e}$ Department of Molecular, Microbial \& Structural Biology, University of Connecticut Health Center, \\ Farmington, CT 06032, USA
}

\author{
* Corresponding author: Associate Professor Graham Nicholson, PhD \\ Neurotoxin Research Group \\ Department of Heath Sciences \\ University of Technology, Sydney \\ PO Box 123, Broadway, NSW, 2007 \\ Australia \\ Tel.: +61-2-9514-2230; fax: +61-2-9514-2228 \\ E-mail address: Graham.Nicholson@uts.edu.au (G.M. Nicholson)
}

\begin{abstract}
Abbreviations: ACh, acetylcholine; ACTX, atracotoxin; BCA, bicinchoninic acid; Bv8, 8kDa protein from the skin secretions of toads (Bombina variegata); CHO, Chinese hamster ovary; DDH, disulfidedirected $\beta$-hairpin; EST, expressed sequence tag; FLIPR, fluorometric imaging plate reader; HEK, human embryonic kidney; ICK, inhibitory cystine-knot; MIT1, mamba intestinal toxin 1 from the venom of the black mamba snake Dendroaspis p. polylepis; PK, prokineticin (also known as endocrine-gland vascular endothelial growth factor or EG-VEGF); PKR, prokineticin receptor; RACE, rapid amplification of cDNA ends; TCEP, Tris(2-carboxyethyl)phosphine; TFA, trifluoroacetic acid.
\end{abstract}

Keywords: ACTX-Hvf17; mamba intestinal toxin 1; Bv8, prokineticin; funnel-web spider

\footnotetext{
${ }^{\dagger}$ Present address. Ludwig Institute for Cancer Research, Courtauld Building, 91 Riding House Street, London, W1W 7BS, United Kingdom
} 


\section{Abstract}

This project identified a novel family of six 66-68 residue peptides from the venom of two Australian funnel-web spiders, Hadronyche sp. 20 and H. infensa: Orchid Beach (Hexathelidae: Atracinae), that appear to undergo N- and/or C-terminal post-translational modifications and conform to an ancestral protein fold. These peptides all show significant amino acid sequence homology to atracotoxin-Hvf17 (ACTX-Hvf17), a non-toxic peptide isolated from the venom of $H$. versuta, and a variety of AVIT family proteins including mamba intestinal toxin 1 (MIT1) and its mammalian and piscine orthologs prokineticin 1 (PK1) and prokineticin 2 (PK2). These AVIT family proteins target prokineticin receptors involved in the sensitization of nociceptors and gastrointestinal smooth muscle activation. Given their sequence homology to MIT1, we have named these spider venom peptides the MIT-like atracotoxin (ACTX) family. Using isolated rat stomach fundus or guinea-pig ileum organ bath preparations we have shown that the prototypical ACTX-Hvf17, at concentrations up to $1 \mu \mathrm{M}$, did not stimulate smooth muscle contractility, nor did it inhibit contractions induced by human PK1 (hPK1). The peptide also lacked activity on other isolated smooth muscle preparations including rat aorta. Furthermore, a FLIPR $\mathrm{Ca}^{2+}$ flux assay using HEK293 cells expressing prokineticin receptors showed that ACTX-Hvf17 fails to activate or block hPK1 or hPK2 receptors. Therefore, while the MIT-like ACTX family appears to adopt the ancestral disulfide-directed $\beta$-hairpin protein fold of MIT1, a motif believed to be shared by other AVIT family peptides, variations in the amino acid sequence and surface charge result in a loss of activity on prokineticin receptors.

\section{Introduction}

Recently a novel non-toxic peptide was isolated from the venom of one of the world's most lethal group of arachnids, the Australian funnel-web spiders (Araneae: Hexathelidae: Atracinae) [42]. This 68-residue protein, ACTX-Hvf17, was isolated from the venom of the Blue Mountains funnel-web spider $H$. versuta. Interestingly, it does not function like classical funnel-web spider atracotoxins to modulate mammalian or insect voltage-gated ion channel function (for reviews see [35, 43]) since it lacks insecticidal activity and fails to affect vas deferens smooth muscle or skeletal muscle contractility. Despite some sequence homology with a variety of colipases it also lacks colipase activity. Accordingly we have been interested in identifying the target of ACTX-Hvf17.

We have recently determined that ACTX-Hvf17 shows significant sequence homology with a variety of novel peptides from fish, frog, snake and several mammalian species belonging to the AVIT family [19] as shown in Fig. 3. This includes MIT1 from the venom of the black mamba snake 
Dendroaspis p. polylepis [2, 39], Bv8 (Bombina variegata $8 \mathrm{kDa}$ protein; [32]) and its orthologs Bm8a-f (B. maxima $8 \mathrm{kDa}$ protein; [4, 21]) from the skin secretions of toads from Bombina spp., and the recently identified prokineticin 1 (PK1; also known as endocrine-gland vascular endothelial growth factor or EGVEGF) and prokineticin 2 (PK2) peptides [27]. These are all 77-94 residue peptides, containing 10 cysteines with conserved spacing, whose N-terminal four residues, 'AVIT', are all identical (Fig. 3). All these peptides, including ACTX-Hvf17, display some limited sequence homology with a variety of colipases but lack colipase activity, are highly resistant to classical specific proteolytic enzymes, and have no overt toxic effects in vertebrates or insects $[19,42]$. ACTX-Hvf17 shares $32 \%$ sequence identity with Bv8, including the 10 conserved cysteine residues, and is $44 \%$ homologous if conservative substitutions are included (Fig. 3). The 3D fold of ACTX-Hvf17 remains undetermined as it is intractable to NMR analysis due to aggregation under a wide variety of conditions. Nevertheless, it is believed that ACTX-Hvf17 adopts the MIT1/colipase fold [42]. Recently two non-AVIT family peptides PRTx16C0 and PRTx16C1 with the highest reported homology to ACTX-Hvf17 have also been isolated from the venom of the Brazilian armed spider Phoneutria reidyi (SWISS-PROT data bank accession numbers P83893 and P83997) (Fig. 3). Unfortunately, like ACTX-Hvf17, no target has yet been identified for these non-toxic arachnid peptides. This is not unique with only a few conotoxins within the venom of marine cone snails (Conus spp.) causing death or overt toxicity, requiring the target of the remaining peptides to be carefully determined.

AVIT family proteins are distributed widely in mammalian tissues and have been shown to cause a variety of actions. These include intestinal contraction [39], hyperalgesia [32, 34], spermatogenesis [48], protection of neuronal cells from apoptosis [31], control of behavioral circadian rhythms [5] and stimulation of endocrine gland angiogenesis [23-26]. In particular MIT1, Bv8 and the prokineticins have all been shown to potently stimulate contraction of guinea-pig ileum smooth muscle with $\mathrm{EC}_{50}$ values in the low to subnanomolar range [27, 32, 39]. However, they do not affect a range of other smooth muscle preparations including vas deferens, trachea, aorta, uterus, and gallbladder. Recent studies have shown that PK1 and PK2 are cognate ligands for two G-protein-coupled receptors designated ZAQ (PK1 receptor; PKR1) and I5E (PK2 receptor: PKR2), respectively [28, 29]. PK1 and PK2 induce a transient increase in intracellular calcium $\left(\left[\mathrm{Ca}^{2+}\right]_{\mathrm{i}}\right)$ with nanomolar potency in Chinese hamster ovary $(\mathrm{CHO})$ cells and human embryonic kidney (HEK293) cells expressing PKR1 and PKR2 receptors [28, 29]. They bind to these receptors with high affinity but PK2 has a higher affinity for both receptors than PK1.

In the present study we have identified six novel MIT-like ACTX orthologs using a combination of HPLC and molecular biology techniques. Given the homology to the AVIT protein family, we hypothesized that ACTX-Hvf17 and these MIT-like spider venom peptides stimulate nociceptors and 
gastrointestinal motility. Thus, these peptides may be useful compounds in the development of prokinetic agents in the treatment of conditions involving poor gastrointestinal motility, or novel analgesics. We show that while ACTX-Hfv17 shares sequence homology to AVIT family proteins and conforms to a similar ancestral protein fold, known as the disulfide-directed $\beta$-hairpin, it lacks the ability to stimulate vertebrate gastrointestinal smooth muscle contractility. It also fails to bind to prokineticin receptors that mediate hyperalgesia and the prokinetic actions on smooth muscle. Modeling of the 3D structure of the MIT-like ACTX family and AVIT family proteins, based on the known structure of MIT1, revealed that the surface charges were also significantly different between these two families of peptides suggesting that the two families may target different receptors and are likely to be functionally distinct.

\section{Materials and methods}

\subsection{Peptide purification}

Venom was collected from both male and female specimens of the Australian funnel-web spider species H. sp. 20 ("Illawarra") and H. versuta, and female specimens of the Orchid Beach variant of $H$. infensa (H. infensa: Orchid Beach) by aspirating venom from the fang-tips (chelicerae) of aggravated spiders into polyethylene pipette tips. Collected venom was dissolved in $0.1 \% \mathrm{TFA}(\mathrm{v} / \mathrm{v})$ for sample transfer to polyethylene tubes and dried down in a Savant SpeedVac for storage at $-20^{\circ} \mathrm{C}$.

ACTX-Hvf17 was isolated from $H$. versuta venom as described previously [42]. Lyophilized crude Hadronyche sp. 20 and H. infensa: Orchid Beach venoms were dissolved in 0.1\% TFA (v/v) and components purified using an analytical C18 RP-HPLC column $(4.6$ x $250 \mathrm{~mm}, 300 \AA$ pore size, $5 \mu \mathrm{m}$ particle size; Vydac, Hesperia, CA, USA). Venom peptides were eluted using an acetonitrile gradient (0$80 \%$ acetonitrile/0.1\% TFA over $80 \mathrm{~min}$ ) at a flow rate of $1 \mathrm{ml} / \mathrm{min}$ governed by a Waters 600 Controller. The UV absorbance was monitored at $214 \mathrm{~nm}$ using a Waters 486 tunable absorbance detector. Purified peptides were collected in polyethylene tubes, lyophilized, and stored at $-20^{\circ} \mathrm{C}$ until required. The molecular mass of each peptide was confirmed by ESI-MS acquired in positive ion mode on a PE-SCIEX API III triple quadrupole mass spectrometer (PE-SCIEX, Canada) equipped with an Ionspray atmospheric pressure ionization source and a Turbospray facility. The full scan data for each venom sample was acquired at an orifice potential of 80 V over a mass range of 400-2100 Da with a step size of 0.2 Da. Data is presented as the average mass in Da.

Toxin quantification was performed using a BCA (bicinchoninic acid) Protein Assay Kit (Pierce, Rockford, IL, USA) using BSA as a standard. Absorbances were read at $570 \mathrm{~nm}$ on a BIO-RAD Model 450 microplate reader. 


\section{$2.2 \mathrm{~N}$-terminal amino acid sequencing}

The disulfide bonds of RP-HPLC purified native peptides were reduced using $100 \mathrm{mM}$ TCEP in 100 $\mathrm{mM}$ ammonium acetate $(\mathrm{pH} 6.5)$ and heating at $45^{\circ} \mathrm{C}$ for $1 \mathrm{~h}$. The reduced cysteine residues were alkylated using $100 \mathrm{mM}$ maleimide/10\% acetonitrile and heating at $45^{\circ} \mathrm{C}$ for $1 \mathrm{~h}$. The reduced and alkylated peptides were isolated by elution on an analytical $\mathrm{C}_{18}$ RP-HPLC column using Vydac $4.6 \mathrm{x}$ $250 \mathrm{~mm}$ (see above) or Zorbax 2.1 x $50 \mathrm{~mm}$ (300 ̊ pore size, $3.5 \mu \mathrm{m}$ particle size or $80 \AA$ pore size, 5 $\mu \mathrm{m}$ particle size; Hewlett Packard, Australia) columns. Partial amino acid sequences of the alkylated peptides were determined using Edman degradation chemistry on an Applied Biosystems 477A Protein Sequencer in conjunction with an Applied Biosystems 120A Analyser (Applied Biosystems/Perkin Elmer, USA). Standard Applied Biosystems sequencing cycles and Perkin Elmer solvents (Applied Biosystems/Perkin Elmer) were used. Data collection and analysis was performed using Model 610A version 2.0 software (Applied Biosystems/Perkin Elmer) on a Macintosh IIsi computer.

\subsection{Enzymic digestion of N-terminally blocked peptide components}

Peptides determined to be $\mathrm{N}$-terminally blocked from preliminary $\mathrm{N}$-terminal amino acid sequencing were fragmented by enzymatic digestion with bovine pancreas trypsin (Sigma, Australia). The peptide fragments were eluted using an analytical RP-HPLC column (Zorbax 2.1 x 50 mm, $80 \AA$ pore size, 5 $\mu \mathrm{m}$ particle size $)$ and an acetonitrile gradient (0-80\% acetonitrile/0.1\% TFA over $80 \mathrm{~min})$ at a flow rate of $200 \mu \mathrm{l} / \mathrm{min}$. The solvent delivery and gradient formation was achieved using a Hewlett Packard Series 1100 HPLC pump system (Hewlett Packard). The UV absorbance of the eluting fragments was monitored at $210 \mathrm{~nm}$ and $280 \mathrm{~nm}$ using a Hewlett Packard Diode Array Detector. Amino acid sequencing was performed on an Applied Biosystems 477A Protein Sequencer.

\section{$2.4 m R N A$ Isolation and $c D N A$ library formation}

The venom glands of anaesthetized mature and juvenile male $H$. sp. 20, and a female H. infensa: Orchid Beach specimens were dissected and the mRNA purified using a Pharmacia Biotech Quickprep Micro mRNA Purification Kit (Pharmacia Biotech, Cleveland, OH). First strand cDNA synthesis from the poly- $\mathrm{A}^{+}$mRNA template of all specimens used a NotI-d(T) $)_{18}$ bifunctional primer $\left\{5^{\prime}-\right.$ AACTGGAAGAATTCGCGGCCGCAGGAAT $_{(18)}$ \} (Pharmacia Biotech) extended with Superscript II Reverse Transcriptase (Invitrogen, Australia). A 5' universal adaptor was added to the single-stranded cDNA (ssDNA) template library of the juvenile male H. sp. 20 and female H. infensa: Orchid Beach 
samples employing the Smart ${ }^{\mathrm{TM}}$ cDNA synthesis technology (Clontech Laboratories, Palo Alto, CA, USA). The juvenile male H. sp. 20 cDNA template library remained single-stranded.

Second strand synthesis of the mature male H. sp. 20 sample employed a Marathon ${ }^{\mathrm{TM}}$ cDNA synthesis kit (Clontech Laboratories) to yield Marathon adaptor double-stranded cDNA (dsDNA). The dsDNA products were cleaned using a BRESAspin PCR Purification Kit (Bresatec, Australia). Second strand synthesis of the female $H$. infensa: Orchid Beach sample was performed by PCR with a Smart ${ }^{\mathrm{TM}}$ adaptor specific primer $\left\{5^{\prime}\right.$-AAGCAGTGGTATCAACGCAGAGT $\}$ and Pfu turbo polymerase (Stratagene, Cedar Creek, TX, USA) following the thermal cycling protocol; 1 cycle of $95^{\circ} \mathrm{C}$ for $1 \mathrm{~min}$, 25 cycles of $95^{\circ} \mathrm{C}$ for $30 \mathrm{~s}, 60^{\circ} \mathrm{C}$ for $30 \mathrm{~s}, 72^{\circ} \mathrm{C}$ for $5 \mathrm{~min}$, and 1 cycle of $72^{\circ} \mathrm{C}$ for $10 \mathrm{~min}$.

\subsection{Primer design and PCR}

5' RACE of the mature male H. sp. 20 dsDNA template using AmpliTaq Gold with GeneAmp kit

(PerkinElmer, Shelton, CT, USA) employed redundant primers 7358-B $\left\{5^{\prime}\right.$-(TC)TC(AGTC)CC(AG)CA(TC)TC(TC)TG(AG)TC(AGTC)CC $\}$ and 7406-B $\left\{5^{\prime}-(\mathrm{AG}) \mathrm{CA}(\mathrm{AGTC}) \mathrm{A}(\mathrm{AG})(\mathrm{AG}) \mathrm{TC}(\mathrm{AG}) \mathrm{TACAT}(\mathrm{TC}) \mathrm{TG}\right\}$ based on the partial amino acid sequences of the mature peptides ACTX-Hs20f7358 and ACTX-Hs20f7406 and a universal adaptor primer (Clontech Laboratories). Thermal cycling followed the cycle protocol; 1 cycle of $95^{\circ} \mathrm{C}$ for $5 \mathrm{~min}, 35$ cycles of $95^{\circ} \mathrm{C}$ for $30 \mathrm{~s}, 50^{\circ} \mathrm{C}$ for $1 \mathrm{~min}, 72^{\circ} \mathrm{C}$ for $1.5 \mathrm{~min}$, and 1 cycle of $72^{\circ} \mathrm{C}$ for $10 \mathrm{~min}$. PCR products were cleaned by agarose gel electrophoresis and then cloned into a pCR 2.1 vector and transformed into host INV $\alpha F^{\prime}$ cells using an Invitrogen TA Cloning Kit (Invitrogen). Sequencing (AGRF, Brisbane, Australia) of the derived leader sequence allowed the gene-specific 3' RACE PCR primers 7358-1A \{5'CGGCCACCCCTGCACTGAACATGT $\}$ and 7406-1A $\left\{5^{\prime}-\right.$ TGCTGAAATTTGTAGTACTGATTTGCC $\}$ to be designed. These gene-specific primers were used in conjunction with a 3' universal primer (Clontech Laboratories) to amplify coding sequences from the cDNA template. Final PCR products ( $\sim 500$ bp) were purified, cloned into a pCR 2.1 vector and transformed into host INV $\alpha$ F' cells using an Invitrogen TA Cloning Kit (Invitrogen), and clones containing the correct insert size were sequenced (AGRF).

\subsection{Expressed sequence tag library}

The PCR products of the female $H$. infensa: Orchid Beach sample were cleaned on a Bresatec PCR purification column, cut with NotI (MBI Fermentas, Hanover, MD, USA), re-cleaned using Bresa-Clean matrix (Bresatec), cloned into pGEM 5Zf(+) Not I/EcoRV dephosphorylated vector (Promega, Australia) 
and transformed into TOP10f' cells (Invitrogen). Clones containing the correct insert size were sequenced (AGRF).

\subsection{Molecular modeling}

The ACTX-Hvf17, ACTX-Hi:OB75212, ACTX-Hs20f7358, Bv8, rPK1 and rPK2 models were constructed using the Homology program within the Insight II molecular modeling system from Accelrys. The amino acid sequences of MIT1 (Boisbouvier et al., 1998), ACTX-Hvf17 (Szeto et al, 2000), ACTX-Hi:OB7512 (this paper), ACTX-Hs20f7358 (this paper), Bv8 [32], rPK1 and rPK2 [29] were aligned within Homology where the alignment was refined using predicted structurally conserved regions of MIT1 defined by PROMOTIF [15]. Refining the models involved repairing splice points such that $\omega$ angles and corresponding bond distances were $180 \pm 1^{\circ}$ and $1.34 \AA$, respectively. The models were then minimized using steepest descents and conjugate gradients taking into account morse and cross terms and charges to a rmsd below $10^{-6} \mathrm{kcal} / \mathrm{mol}-\AA \AA$. Finally, the models were validated using the programs Profiles 3D (within the Homology program) and Procheck [33].

\subsection{Isolated smooth muscle preparations}

All animal experimentation was approved by the Animal Care and Ethics Committees of the University of Technology, Sydney and Monash University. Given the lack of H. sp. 20 and H. infensa: Orchid Beach spiders and thus sufficient venom for pharmacological investigations, experiments were only carried out with ACTX-Hvf17.

\subsubsection{Isolated guinea-pig ileum smooth muscle preparation}

Dunkin-Hartley guinea-pigs (0.5-1.3 kg), of either sex, were sacrificed by dislocation of cervical vertebrae. Segments of ileum $(2-3 \mathrm{~cm})$, dissected $10 \mathrm{~cm}$ distal to the ileo-caecal junction, were then dissected and attached to electrodes. These were mounted in $5 \mathrm{ml}$ isolated organ baths in a Krebs solution of the following composition (mM): $\mathrm{NaCl} 118.4, \mathrm{KCl} 4.7, \mathrm{MgSO}_{4} 1.2, \mathrm{KH}_{2} \mathrm{PO}_{4} 1.2, \mathrm{CaCl}_{2} 2.5$, $\mathrm{NaHCO}_{3} 25.0$ and D-glucose, 11.1. The solution was maintained at $34^{\circ} \mathrm{C}$ and bubbled with carbogen $\left(95 \% \mathrm{O}_{2}\right.$ and $\left.5 \% \mathrm{CO}_{2}\right)$. For electrically stimulated preparations, ilea were transmurally stimulated by regular field pulses $(40 \mathrm{~V}, 0.5 \mathrm{~ms}, 0.2 \mathrm{~Hz})$ using a Grass $\mathrm{S} 88$ stimulator.

\subsubsection{Isolated rat stomach fundus and aorta smooth muscle preparations}

Adult male Wistar rats (300-350 g) were sacrificed by dislocation of cervical vertebrae. The stomach and aorta were removed and placed in a Krebs solution of the following composition (mM): $\mathrm{NaCl} 119$, 
$\mathrm{KCl}$ 4.7, $\mathrm{MgSO}_{4}$ 1.0, $\mathrm{CaCl}_{2}$ 2.5, $\mathrm{NaHCO}_{3}$ 25.0, $\mathrm{KH}_{2} \mathrm{PO}_{4} 1.2$ and D-glucose 11.1. The solution was maintained at $37^{\circ} \mathrm{C}$ and bubbled with carbogen $\left(95 \% \mathrm{O}_{2}\right.$ and $\left.5 \% \mathrm{CO}_{2}\right)$. The fundus portion of the rat stomach was divided in half by making a midline incision along the greater curvature. The mucosa was carefully excised. A medial longitudinal strip $(3 \times 15 \mathrm{~mm})$ was obtained from each half of the divided fundus by making cuts parallel to the midline incision. Rat aortas were removed from the section of the thoracic aorta between the aortic arch and the diaphragm. Spiral strips $(2 \times 15 \mathrm{~mm})$ were obtained from the aorta and cleaned of adhering fat and connective tissue. Tissues were then placed in $10 \mathrm{ml}$ organ baths.

\subsubsection{Contractility studies}

Isometric muscle tension was recorded with a force-displacement transducer (Nihon Kohden SB-1T, Tokyo, Japan) on a polygraph (Nihon Kohden) or a Grass transducer (FTO3) connected to a Grass polygraph (Model 79D). Data were also digitized using a MacLab 4/s or Powerlab/400 data acquisition system (ADInstruments, Castle Hill, Sydney, Australia). Each preparation was placed under an initial resting tension of $1 \mathrm{~g}$ and allowed to equilibrate for $1 \mathrm{~h}$ before experimental procedures were carried out. Muscle tension in each tissue was recorded in the absence of additives or following injection of peptides (5-20 $\mu 1$ in water) directly into the bath buffer. To confirm stable contractions of sufficient amplitude, contractions of smooth muscle preparations to $1 \mu \mathrm{M}$ ACh (ileum and fundus) or $50 \mathrm{mM} \mathrm{KCl}$ (aorta) were checked before addition of ACTX-Hvf17 or vehicle, and at the conclusion of the experiment. To determine if ACTX-Hvf17 blocked contractions mediated via prokineticin receptors, responses to recombinant hPK1 (Peprotech, Princeton, NJ, USA) were measured before, and following, addition of ACTX-Hvf17.

\subsection{Calcium-mobilization assay using a fluorometric imaging plate reader (FLIPR)}

Assessment of ACTX-Hvf17 function on prokineticin receptors was accomplished using a fluorometric imaging plate reader assay (FLIPR, Molecular Devices, Sunnyvale, CA, USA). Briefly, 24 h prior to experimentation, HEK293 cells stably expressing human prokineticin 1 (hPKR1) or human prokineticin 2 (hPKR2) receptors were plated into clear bottom, black-walled 96-well plates precoated with poly-D-lysine (Becton-Dickinson, Franklin Lakes, NJ, USA) at a density of 5 x $10^{5}$ cells per well. The cDNAs encoding hPKR1 and hPKR2 receptors were obtained by PCR from genomic DNA using primers based on the publicly available sequences (GenBank accession numbers AF506287 and AF506288, respectively). The cDNAs were expressed from the pCDNA3.1 vector (Invitrogen, Carlsbad, CA, USA). On the day of screening, cells were loaded using the calcium 3 assay kit (Molecular Devices) 
according to the manufacturer's protocol. ACTX-Hvf17 was added at the indicated concentrations and the elevation in fluorescence, in proportion to an increase in $\left[\mathrm{Ca}^{2+}\right]_{i}$, was monitored. In cases where ACTX-Hvf17 was tested for antagonist activity the initial exposure to the spider peptide was followed five minutes later by the introduction of $10 \mathrm{nM}$ recombinant hPK1 (Peprotech).

\subsection{Chemicals}

Unless otherwise stated, all chemicals were of analytical grade and were purchased from Sigma-Aldrich Chemical Co. (St Louis, MO, U.S.A.).

\subsection{Statistical analysis}

Numerical data are presented as the mean \pm S.E. ( $n$, number of observations). Statistical differences were determined using a Student's paired t-test, at $P<0.05$.

\section{Results}

\subsection{Isolation of a novel family of spider peptides with sequence homology to the MIT-like ACTX-Hvf17}

Components of H. sp. 20 ("Illawarra") funnel-web spider venom with $\mathrm{M}_{\mathrm{r}} \sim 7400$ Da were sequenced employing Edman degradation in conjunction with molecular biology techniques. Initially, three peptides, with $\mathrm{M}_{\mathrm{r}}$ of 7357.8, 7406.2 and 7390.0 Da, were isolated from the venom by RP-HPLC (Fig. 1A) and subjected to automated N-terminal amino acid sequencing. An additional small peak with a $\mathrm{M}_{\mathrm{r}}$ of 7496.2 Da was also noted in the HPLC chromatograms but not subjected to N-terminal amino acid sequencing. The peptide component with a $\mathrm{M}_{\mathrm{r}}$ of 7357.8 Da yielded a partial 37-residue N-terminal sequence. This sequence information displayed significant homology to the MIT-like molecule ACTX-Hvfl7 from the venom of $H$. versuta (Fig. 2B). This MIT-like atracotoxin molecule was named ACTX-Hs20f7358 following the nomenclature system for atracotoxins (ACTX, [11]), where the suffix 'Hs20' represents the Genus and species name and ' $\mathrm{f} 7358$ ' represents the venom HPLC fraction with $\mathrm{M}_{\mathrm{r}}$ 7357.8. However, the remaining two components with $\mathrm{M}_{\mathrm{r}}$ of 7406.2 and $7390.0 \mathrm{Da}$ failed to yield any sequence information suggesting that both molecules are blocked at the N-terminus or possess a cyclic peptide backbone. Subsequently, the fraction with a $\mathrm{M}_{\mathrm{r}}$ of $7406.2 \mathrm{Da}$ was subjected to enzymatic digestion with trypsin. Two resulting fragments were isolated by RP-HPLC and sequenced by automated N-terminal amino acid sequencing. Only one digestion fragment sequenced successfully and yielded an eight-residue sequence LSQMYDLC showing homology to residues 25-32 in ACTX-Hvf17 (Fig. 3) and ACTX-Hs20f7358 (Fig. 2B \& 3). This fraction was therefore named ACTX-Hs20f7406.

Based on the retention time and molecular weight similarities, the molecule with a $\mathrm{M}_{\mathrm{r}}$ of $7390.0 \mathrm{Da}$ 
was predicted to be an isoform of the 7406.2 Da component. As a result it was anticipated that both components would be identified from the use of a single 3' RACE primer. Due to the expected sequence characterisation of both components in the molecular biology experiments, enzymatic digestion was not performed on the 7390.0 Da fraction.

\subsection{Sequence identification of MIT-like ACTX peptides using RACE}

A cDNA library was used in the identification of these Hadronyche sp. 20 ("Illawarra") venom components. A redundant 5' RACE primer (7406-B) was constructed based on the partial N-terminal sequence information obtained from the tryptic digest fragment of ACTX-Hs20f7406. PCR experiments of the male H. sp. 20 cDNA template library with the 5' RACE primer generated a DNA product of approximately 210-250 bp in length. The cloning and subsequent sequencing of three clones of products derived from the H. sp. 20 cDNA library yielded N-terminal sequence information of the mature peptide and a short leader sequence. The short leader revealed evidence of a $54 \mathrm{bp}$ signal peptide, but no evidence of a propeptide sequence. The absence of a propeptide sequence is in contrast to other nucleotide sequences identified from the venom ducts of Australian funnel-web spider species [41].

PCR experiments performed on the H. sp. 20 cDNA template library with a second 5' RACE primer (7358-B) designed on the partial amino acid sequence obtained for ACTX-Hs20f7358 provided a DNA product of approximately 160-200 bp in length. Cloning and subsequent sequencing provided similar leader sequence information to that obtained for ACTX-Hs20f7406, but differed by $6 \mathrm{bp}$.

The combined leader sequence information derived from the 5' RACE experiments on the H. sp. 20 cDNA template library was used to design two gene specific 3' RACE primers (7406-1A and 7358-1A) to allow determination of the complete coding sequences of $M_{r} 7357.8, M_{r} 7406.2$ and $M_{r} 7390.0$. Cloning and subsequent sequencing of multiple clones of the corresponding DNA products derived from the H. sp. 20 cDNA template library identified five MIT-like molecules. The amino acid sequences of the five identified molecules show homology to the peptide ACTX-Hvf17 isolated from the venom of Hadronyche versuta [42]. The cDNA sequences derived for the MIT-like components from the H. sp. 20 library consist of coding sequences of $267 \mathrm{bp}$ and $252 \mathrm{bp}$ translating to 89 and 84 amino acids respectively. The 7406-1A gene specific 3' RACE primer was designed to maximize the number of base pair differences between the ACTX-Hs20f7358 and ACTX-Hs20f7406 leader sequences to allow differential selection of the two sequences by variation of the annealing temperature during PCR. The 5' end of the primer corresponds to the $\mathrm{T}$ of the translation start codon (ATG - coding for Met) and as such nucleotide sequences obtained from this primer have an incomplete start codon. Since it is known the first two nucleotides of sequences obtained from the 7406-1A primer are part of the start codon, the 
amino acid sequences are taken to include Met for the sake of completeness. The predicted mature peptide sequences comprise 71 and 66 amino acids respectively and contain a 10 cysteine framework forming five disulfide bonds.

\subsection{Post-translational C-terminal processing and N-terminal block of MIT-like ACTX}

Examination of the mature peptide sequences derived from the 7406-1A 3' RACE primers revealed theoretical calculated molecular weights of 7779.5, 7795.5, 7807.6, 7358.1 and 7868.8 Da, when accounting for the presence of five disulfide bonds. The presence of the molecule ACTX-Hs20f7358 (GenBank accession number AY914165) was easily identified in the RP-HPLC/ESI-MS data (Fig. 1A) and the sequence obtained matched the partial sequence information collected from N-terminal amino acid sequencing of native material. No molecules with molecular weights of 7779.5, 7795.5, 7807.6 or 7868.8 Da were identified in the RP-HPLC/ESI-MS profile of the H. sp. 20 venom. Further observation of the sequences predicted that the discrepancy in molecular weight was the result of a post-translational cleavage of the C-terminal residues, Arg-Ser-Glu. Post-translational C-terminal "trimming" has been reported for a number of peptide toxin components, including spider [9, 17], bee [20], cone snail [6] and scorpion [3] toxins. The respective molecular weights of the four components following removal of the C-terminal fragment (RSE) are 7407.1, 7423.1, 7435.2 and 7496.4 Da. A molecule of molecular weight 7496.2 Da (ACTX-Hs20f7496, GenBank accession number AY914164) is evident in the RP-HPLC/ESIMS data (Fig. 1A) and elutes close to the two components with $\mathrm{M}_{\mathrm{r}}$ of 7406.2 and $7390.0 \mathrm{Da}$.

The failure of the N-terminal amino acid sequencing experiments performed on the two molecules with $\mathrm{M}_{\mathrm{r}}$ of 7406.2 and $7390.0 \mathrm{Da}$ is most likely accounted for by the presence of N-terminal Gln residues. The three 7407.1, 7423.1 and 7435.2 Da components have an N-terminal Gln, a residue known to commonly form pyroglutamate and block N-terminal sequencing. The formation of pyroglutamate from Gln results in the loss of $\mathrm{NH}_{3}$ corresponding to a mass of $17 \mathrm{Da}$. A further recalculation of the masses of the three components, allowing for the C-terminal trimming and pyroglutamate formation, reveals respective molecular weights of $7390.1 \mathrm{Da}$ (ACTX-Hs20f7390, GenBank accession number AY914166), 7406.1 Da (ACTX-Hs20f7406, GenBank accession number AY914168) and 7418.1 Da (ACTX-Hs20f7418, GenBank accession number AY914167). The two components ACTX-Hs20f7390 and ACTX-Hs20f7406 were easily identified in the RP-HPLC/ESI-MS data (Fig. 1A), however the third component ACTX-Hs20f7418 was not evident. It is possible that the component ACTX-Hs20f7418 is expressed at levels below the level of detection on the mass spectrometer and as a result is not evident in the RP-HPLC/ESI-MS data. 
3.4 The Expressed Sequence Tag (EST) technique identified the presence of a MIT-like ACTX in the venom of Hadronyche infensa: Orchid Beach.

During the course of the studies on MIT-like ACTX components, a further component belonging to this family, ACTX-Hi:OBf7512 (GenBank accession number AY914163), was identified from the venom glands of a female Hadronyche infensa: Orchid Beach specimen using Expressed Sequence Tag (EST) techniques (Fig. 2A). The sequence information provided a mature peptide sequence containing an identical C-terminal tail (Arg-Ser-Glu) to some of the sequences of this family derived from the RACE experiments (Fig. 2B). It was anticipated, based on molecular weight calculations, that these Cterminal residues were post-translationally cleaved to produce the mature product.

The calculated theoretical molecular weight of the fully cleaved mature product, accounting for the formation of five disulfide bonds and C-terminal tail cleavage, was 7586.5 Da. This molecular weight is not observed in the RP-HPLC/ESI-MS data, however the sequence does contain an N-terminal Gln residue that, based on the ACTX-Hs20 molecules identified, is predicted to form pyroglutamate. A recalculation of the molecular weight, allowing for pyroglutamate formation, arrived at a molecular weight of $7569.5 \mathrm{Da}$. No molecules of this mass were observed in the Hadronyche infensa: Orchid Beach venom profile. It was noted that the mature sequence, following removal of the Arg-Ser-Glu Cterminal tail, was one residue (Gly) longer than the other sequences predicted to involve identical Cterminal trimming. Furthermore, cleavage of the C-terminal Gly residue from other toxins has been reported to form a C-terminal amide $[3,6,20]$. Removal of the Gly results in a molecular weight of 7512.4 Da, and a peak corresponding to this molecular weight is evident in the RP-HPLC/ESI-MS chromatogram labeled ACTX-Hi:OBf7512 (Fig. 1B). It is also possible that the cleavage of the Gly forms a C-terminal amide, to yield a component having an $\mathrm{M}_{\mathrm{r}}$ of 7511.5 Da. Precision mass data was not collected on native material of the component and therefore the exact molecular weight and the possibility of a C-terminal amide has not been conclusively resolved.

A comparison of the MIT-like ACTXs revealed that these molecules constitute two groups. The first of these groups consists of the 66-residue peptide, ACTX-Hs20f7358, which does not undergo any primary sequence post-translational modifications. The second group is composed of the 68-residue components, ACTX-Hvf17 and its ACTX orthologs -Hs20f7406, -Hs20f7390, -Hs20f7418, -Hs20f7496, and $\mathrm{Hi}: \mathrm{OBf7512}$, and demonstrates post-translational C-terminal trimming of a propeptide to yield the mature product (although this is not known for ACTX-Hvf17). A blastp search of the SwissProt/TrEMBL knowledgebase revealed high sequence homology of the MIT-like ACTX with ACTX-Hvf17, two non-toxic spider peptides from the venom of the spider P. reidyi, and to a lesser extent, AVIT family proteins (Fig 3). 


\subsection{Effect of ACTX-Hvf17 on gastrointestinal and vascular smooth muscle}

Previously, ACTX-Hvf17 has been screened for mammalian bioactivity using a rat vas deferens bioassay [42]. In this previous study it was found that the peptide lacked any observable activity in this smooth muscle preparation. Given the potential activity of the peptide on other smooth muscle preparations, particularly gastrointestinal tissue, the present study therefore tested the activity of ACTXHvf17 on isolated ileum and stomach fundus, as well as aorta. As shown in Fig. 4, ACTX-Hvf17 at concentrations up to $1 \mu \mathrm{M}$ had no effect on gastrointestinal smooth muscle. This was evidenced by a lack of change in resting (baseline) tension of isolated guinea-pig ileum longitudinal muscle $(n=3$; Fig. $4 \mathrm{Ab})$ and rat stomach fundic muscle strip preparations ( $n=6$; Fig. 4B). In addition, ACTX-Hvf17 failed to alter stimulated contractions of guinea-pig ileum $(n=3$; Fig. 4Aa). Furthermore, ACTX-Hvf17 (1 $\mu \mathrm{M})$ failed to affect the resting tension of isolated aortic smooth muscle preparations $(n=3$, Fig. $4 \mathrm{C})$ indicating that the peptide does not directly stimulate vascular smooth muscle.

To investigate the possible antagonism of prokineticin receptors by ACTX-Hvf17, smooth muscle strips from rat stomach fundus and guinea-pig ileum were contracted with hPK1 and ACTX-Hvf17 added $10 \mathrm{~min}$ before a second challenge of hPK1. At concentrations of hPK1 up to $10 \mathrm{nM}$, hPK1 only caused contractions in 3 out of 6 fundic strips. The contractile responses in these 3 tissue strips were only around $20 \%$ of the responses elicited by $1 \mu \mathrm{M}$ ACh. In addition, following the first application of hPK1, a strong tachyphylaxis developed such that additional applications of hPK1 did not result in any contraction. It was thus not possible to obtain a reproducible response to hPK1 in these preparations. This strong tachyphylaxis supports the observation by Mollay et al. (1999) who demonstrated that an intense tachyphylaxis developed after the first exposure of MIT1 and Bv8 to jejunum and colon. In contrast, hPK1 produced reproducible dose-dependent responses in guinea-pig ileum. However, concentrations of ACTX-Hvf17 up to $1 \mu \mathrm{M}$ failed to alter the amplitude of these contractions, signifying that the peptide does not block prokineticin receptors in guinea-pig ileum $(P>0.9, n=3$; Fig. 4Ac).

\subsection{Effects of ACTX-Hvf17 on expressed prokineticin receptors}

To directly evaluate the effect of ACTX-Hvf17 on prokineticin receptors, we assessed the increase in $\left[\mathrm{Ca}^{2+}\right]_{\mathrm{i}}$ in HEK293 cells stably expressing hPKR1 or hPKR2 receptors using a fluorometric imaging plate reader (FLIPR) assay. In this assay hPK1 (Fig. 5A) caused a dose-dependent transient increase in $\left[\mathrm{Ca}^{2+}\right]_{\mathrm{i}}$. Initial experiments were designed to identify any potential agonist activity. As with the isolated smooth muscle preparations, concentrations of ACTX-Hvf17 up to $100 \mathrm{nM}$ failed to evoke an increase in $\left[\mathrm{Ca}^{2+}\right]_{\mathrm{i}}$ in cells expressing either hPKR1 (Fig. 5A) or hPKR2 receptors (data not shown). 
Consequently, we examined the antagonist activity of ACTX-Hvf17 on prokineticin-induced increases in $\left[\mathrm{Ca}^{2+}\right]_{\mathrm{i}}$. Cells expressing hPKR1 or hPKR2 receptors were incubated with $10 \mathrm{nM}$ hPK1 together with increasing concentrations of ACTX-Hvf17. Concentrations of ACTX-Hvf17 as high as $100 \mathrm{nM}$ failed to inhibit hPK1-induced transient increases in $\left[\mathrm{Ca}^{2+}\right]_{\mathrm{i}}$ (Fig. 5B). These data indicate that ACTX-Hvf17 lacks both agonist and antagonist activity at hPKR1 and hPKR2 receptors.

\subsection{Molecular modeling of MIT-like ACTX}

The 3D structures of the MIT-like ACTXs, Bv8, PK1 or PK2 have not yet been solved. ACTXHvf17 aggregates under a wide variety of solvent conditions at concentrations sufficient for NMR making NMR structural analysis futile [42], whereas structural analysis on the other peptides has not yet been attempted. However, the 3D structure of MIT1 has been determined (Fig. 6A) and despite a low level of sequence homology, was found to be very similar to porcine colipase [2, 45]. Given the overall sequence homology between MIT-like ACTX, Bv8, PK1, PK2, MIT1 and to a lesser extent colipase, including the conserved cysteine residues and resistance to proteolytic digestion [2], MIT-like ACTX, Bv8, PK1 and PK2 were proposed to have the same 3D folds as MIT1 and colipase [42]. Modeling of the ACTX-Hvf17, ACTX-Hi:OBf7512, ACTX-Hs20f7358, Bv8, rPK1 and rPK2 structures based on MIT1, as shown overlaid in Fig. 6B (ACTX-Hvf17 only), reveals that the structurally conserved regions of the putative 3D structure of ACTX-Hvf17, ACTX-Hi:OBf7512, ACTX-Hs20f7358, Bv8, rPK1 and rPK2 are indeed very similar to those of the MIT1 and colipase peptides. This is not surprising given that all 10 cysteine residues align closely between the eight peptides even though the primary sequence is not identical in length. Additionally, loop 3 in all peptides aligns well to MIT1 having an rmsd over

the backbone atoms of approximately $0.36 \AA$. However, models displaying the electrostatic surface potential revealed that MIT-like ACTX peptides do not share similar surface charge distribution as AVIT family proteins (Fig. 6C-D). In addition, while the AVIT family proteins share a comparable surface polarity distribution the MIT-like ACTX peptides do not (Fig. 6C-D).

\subsection{ACTX-Hvf17 conforms to the DDH ancestral protein fold}

Previously, MIT1, colipase, and a wide variety of other eukaryotic proteins, have been characterized as comprising a disulfide-directed $\beta$-hairpin (DDH) fold [47], as shown in Fig. 7Aa. The amino acid consensus sequence of the $\mathrm{DDH}$ fold can be written as $\mathrm{CX}_{5-9} \mathrm{CX}_{2}[\mathrm{G}$ or $\mathrm{P}] \mathrm{X}_{2} \mathrm{CX}_{6-19} \mathrm{C}$, where $\mathrm{X}$ is any amino acid [47]. A strictly conserved central Gly or Pro is also present in loop 3, which ensures a tight turn prior to the first $\beta$-stand (Fig. 6A-B). In general, hydrophobic residues also tend to follow the central Gly/Pro in loop 3. The inhibitor cystine-knot (ICK) motif present in all ACTX structures 
determined to date appears to have evolved from this simpler ancestral fold [47]. In contrast to the ICK motif, which has not been found in vertebrates and includes a triple-stranded antiparallel $\beta$-sheet (Fig. $7 \mathrm{Ab})$, the DDH fold is found in a variety of eukaryotes, has no requirement for an N-terminal $\beta$-strand, and only two mandatory disulfide bridges form the bulk of the hydrophobic core of this domain. Importantly, we observed a homologous DDH domain in all the MIT-like ACTX and the P. reidyi spider peptides PRTx16C0 and PRTx16C1 (Fig. 7B). In addition, there is a strong conservation of residues in loop 3 between the MIT-like ACTX, the P. reidyi spider peptides and the AVIT family of proteins with a consensus sequence of CGXGXCC, where $\mathrm{X}$ is any amino acid. Therefore it appears that MIT-like ACTXs, PRTx16C0, PRTx16C1, Bv8, MIT1, and the various prokineticins may have arisen from simple gene duplication followed by minor residue changes in the intervening loops (Fig. 7B). In the case of MIT-like ACTXs and the P. reidyi peptides, however, they appear to have undergone the greatest alteration or deletion of loop residues, particularly in the N-terminal and loop 4 regions, resulting in loss of activity on prokineticin receptors.

The DDH motif of the MIT-like ACTX differs from other DDH members by having only four residues following the first cysteine in the amino acid consensus sequence. Furthermore, huwentoxin-II (Shu et al., 2002), another spider peptide conforming to the DDH fold, only has four residues preceding the last cysteine in the amino acid consensus sequence (Fig. 7B). Hence the consensus sequence needs to be modified to $\mathrm{CX}_{4-19} \mathrm{CX}_{2}[\mathrm{G}$ or $\mathrm{P}] \mathrm{X}_{2} \mathrm{CX}_{4-19} \mathrm{C}$. However, the presence of five residues in loop 3 with a central Gly residue in virtually all cases, exclude the possibility that the MIT-like ACTXs adopt the cystine-stabilized $\beta$-sheet (CSB) motif [14]. In the CSB fold the number of residues in loop 3 can be as low as two and there is no requirement for a central glycine residue.

\section{Discussion}

Through a combined approach of amino acid sequencing and molecular biology techniques, five homologous components were isolated from the venom of H. sp. 20. An additional ortholog was also identified from the venom of $H$. infensa: Orchid Beach using EST molecular biology techniques. With the exception of the component ACTX-Hs20f7418 from H. sp. 20 venom, all molecules were accounted for in their respective venom RP-HPLC/ESI-MS profiles (Fig. 1). This family of peptides shows high amino acid sequence homology with each other particularly in the leader sequence (Fig. 2B). In addition, they all show high sequence homology with ACTX-Hvf17 from $H$. versuta venom [42] and to a lesser extent PRTx16C0 and PRTx16C1 from the venom of the spider P. reidyi. Like ACTX-Hvf17, the target of these peptides and those from $P$. reidyi venom is yet unknown.

ACTX-Hvf17, and the orthologous peptides identified in the present study, also show significant 
sequence homology with MIT1. Hence we have termed this group of non-toxic atracotoxins the 'MITlike' ACTX family. MIT1 belongs to the 'AVIT' protein family including Bv8 and orthologs from the PK1/EG-VEGF and PK2 families [19]. All AVIT family members that have been tested so far stimulate contraction of fundus and ileum smooth muscle via interaction with prokineticin receptors. Therefore, we determined if MIT-like ACTX interacted with prokineticin receptors to increase gastrointestinal smooth muscle contractility.

Unlike AVIT proteins, this study showed that the prototypical MIT-like ACTX, ACTX-Hvf17, does not stimulate gastrointestinal smooth muscle contractility as evidenced by a lack of activity on stimulated guinea-pig ileum and rat stomach fundus. Neither did the peptide affect responses of ileum smooth muscle to ACh. In addition, the peptide failed to contract other smooth muscle preparations including rat aorta. We also assessed the ability of ACTX-Hvf17 to act as a prokineticin receptor antagonist by determining the ability of the peptide to inhibit hPK1-induced contractions of gastrointestinal smooth muscle. We were unable to assess the effects on hPK1-induced contractility in fundus and aorta due to the inconsistent response and strong tachyphylaxis of hPK1 in these tissues. Nevertheless, ileal muscle contractions elicited by 4 nM hPK1 were unaffected by $1 \mu \mathrm{M}$ ACTX-Hvf17.

In addition to experiments performed on isolated smooth muscle, we also investigated the effects of ACTX-Hvf17 on hPKR1 and hPKR2 receptors expressed in HEK293 cells. These are orphan G-proteincoupled receptors that are present on various mammalian cells and were found to bind AVIT family proteins. Receptor activation leads to an increase in $\left[\mathrm{Ca}^{2+}\right]_{i}$, as well as an increased phosphoinositide turnover, and activation of the p44/p42 mitogen-activated protein kinase signaling pathway (for a review see [19]). These actions are consistent with the effects of prokineticins on smooth muscle contractility and angiogenesis. Using a FLIPR assay, ACTX-Hvf17 at concentrations up to $100 \mathrm{nM}$ failed to cause an increase in $\left[\mathrm{Ca}^{2+}\right]_{\mathrm{i}}$ indicating that the peptide lacks agonist activity on both hPKR1 and hPKR2 receptors. In addition, increasing concentrations of ACTX-Hfv17 up to $100 \mathrm{nM}$ failed to inhibit the hPK1-induced increase in $\left[\mathrm{Ca}^{2+}\right]_{\mathrm{i}}$ signifying that ACTX-Hf17 lacks antagonist activity on both PKR1 and PKR2 receptors. In concert, the results from the FLIPR assay and isolated smooth muscle preparations indicate that ACTX-Hvf17 does not bind to either PKR1 or PKR2 receptors despite sequence homology to the AVIT family proteins. The PK2 protein is not commercially available so we were unable to test this protein in these assays. It therefore remains a possibility that ACTX-Hfv17 can block the PK2 interaction with the receptors, although this is unlikely given the overlap in efficacy of PK1 and PK2 at prokineticin receptors. It is also possible that prokineticin receptors exist in prey species that differ significantly from those found in vertebrates and that the MIT-like peptides show phyla-specificity for these targets. Nevertheless no overt toxicity or behavioural changes were noted following injection of 
ACTX-Hvfl7 into crickets, and thus this is improbable.

Modeling of the electrostatic surface potential of the MIT-like ACTX family based on the known structure of MIT1 reveals that the surface charge distribution is distinct from the AVIT family. Indeed, while the surface charge distribution within the AVIT family appear to be comparatively similar, the electrostatic surface maps of the MIT-like ACTX family appear quite inconsistent between members. This adds further support to the finding that the MIT-like ACTX fail to bind to PKR1 or PKR2 receptors. Interestingly, one notable sequence characteristic of these MIT-like arachnid venom peptides is that they all appear to lack the characteristic N-terminal AVITG[A or V]C sequence of AVIT proteins, suggesting a functional role for this region. Furthermore, there is considerable lack of sequence homology in loop 4 (e.g. Ser ${ }^{15}$-His $^{21}$ in ACTX-Hvf17). Unfortunately little is known about the structure-function relationships of AVIT protein family members, however it is unlikely that the C-terminal domain plays a significant role in the activity of this family of peptides since there is considerable sequence variability in the $\mathrm{C}$-termini beyond the last Cys residue. It is also unlikely that the residues in loop 3 are important for activity given the high homology with the inactive ACTX-Hvf17 peptide. Experiments with Nterminal truncates could therefore help to address any functional importance of the N-terminus, especially the first five to six residues, of the AVIT family of proteins.

Given the likelihood that these venom peptides and the AVIT proteins share the ancestral DDH fold motif it is conceivable that the two groups, and other proteins such as colipase, evolved from a very ancient ancestor. However, following the period of the chordate-arthropod divergence around 1000 million years ago [46], the mutations in the primary sequence, possibly in the N-terminus and/or loop 4, resulted in the loss of activity on prokineticin receptors. These could be due to positive selection for adaptive changes as part of a general evolution of the gastroenteropanceatic hormonal system in mammals. That is why the functional constraints for the AVIT proteins are practically unchanged in all lineages. Nevertheless, an alternative theory is that spider peptides and AVIT proteins evolved separately. Given that the DDH fold is present in plants (e.g. gurmarin) as well as invertebrates (e.g. spider venom peptides) it can be argued that they evolved independently rather than from a pancreatic origin as suggested by Boisbouvier et al. (1998). In support ACTX-Hvf17, colipases, and MIT1 are highly resistant to proteases and convergent evolution may have arisen because the DDH fold is an efficient way to construct a tight globular protein that is resistant to proteolysis. This has been as previously argued for the more elaborate, but related, ICK motif [10] (Fig. 7Ab).

Based on the partial N-terminal sequencing data of ACTX-Hs20f7358 and the published sequence of ACTX-Hvf17 [42], cleavage of the mature peptides from the leader sequences is expected to occur following the residues Ser-Ala for the components ACTX-Hs20f7418, ACTX-Hs20f7406 and ACTX- 
Hs20f7390 and residues Phe-Ala for molecules ACTX-Hi:OBf7512, ACTX-Hs20f7358 and ACTXHs20f7496 (Fig 2B-C). These cleavage sites are different to the "classical" prohormone cleavage recognition site Lys-Arg between a propeptide and mature-peptide [38], but are consistent with the signal peptide cleavage sites predicted using SignalP (v3.0) [1] and those of the AVIT peptide family $[32,48]$. Importantly, this leader sequence configuration is unlike other neurotoxic ACTX belonging to the $\delta-1, \omega-1, \omega-2$ and $\kappa-1$ ACTX families [41], where the leader sequence comprises of a signal sequence followed by a propeptide, with the propeptide being cleaved to form the mature-peptide. Although no literature reports of mature-peptide cleavage following the residues Ser-Ala were found, cleavage of the mature-peptide from the leader sequence for the spider venom peptide latrodectin from the Mediterranean black widow spider (Latrodectus tredecimguttatus) was found to occur following the residues Phe-Ala [37]. The latrodectin leader sequence is identical in length and similar in sequence to the leader sequence of the MIT-like ACTX family; 28\% identity increasing to 67-78\% homology if conservative substitutions are included. Numerous examples of leader sequences composing only a signal peptide have also been reported for scorpion toxins [3]. Despite the homology shared in the leader sequences, the mature MIT-like ACTX peptide sequences have no notable homology with the mature latrodectin sequence. It is also notable that, like the MIT-like ACTX family, no functional activity has been determined for latrodectin. The leader sequences of Bv8 and its mammalian orthologs hBv8 and mBv8 also lack a propeptide region and display some sequence similarity with the leader sequences determined for MIT-like ACTX peptides, however they are more variable than the leader sequence for latrodectin (17-22\% identity, and 39-56\% similarity if conservative substitutions are included)[32, 48].

All the novel peptide components, except ACTX-Hs20f7358, were concluded to undergo post-translational modifications involving $\mathrm{N}$-terminal pyroglutamate formation and/or C-terminal “trimming”. Despite this not being reported for other ACTX peptides, post-translational pyroglutamate formation has been described with contulakin-G from the cone snail Conus geographus [7], several members of the charybdotoxin-like $\alpha-K T x ~ 1$ subfamily of scorpion toxins (for a review see [44]) and dendrotoxin from the mamba Dendroaspis augusticeps [8]. C-terminal trimming has also been reported for a number of peptide toxin components, including the spider toxins Tx1, Tx3-2 and Pn3A from the Brazilian armed spider $P$. nigriventer [9, 17], the bee toxin melittin [20], $\omega$-CTX GVIA from $C$. geographus [6] and the scorpion toxins AaHI, AaI', AaHII and AaHIII [3] from the scorpion Androctonus australis hector.

In conclusion, we report the identification of a group of novel atracotoxin peptides, the MIT-like ACTX family, with novel leader sequence structure that appear to undergo post-translational modification. The results of the present study also illustrate that the prototypical MIT-like atracotoxin, 
ACTX-Hvf17, does not increase smooth muscle contractility. This finding, coupled with a previous report that it does not act as a colipase [42], suggest that the peptide and other MIT-like ACTXs are not involved in prey digestion. In addition, the finding that the peptide fails to interact with prokineticin receptors, targeted by AVIT family proteins negates the possibility that MIT-like ACTX act as hyperalgesic agents to sensitize nociceptors and increase pain in prey species [32, 34]. Modeling of the 3D structure of the MIT-like ACTX and AVIT family proteins also indicates that these two groups do not share similar surface distribution, further supporting the finding that MIT-like ACTX lack affinity for prokineticin receptors. Therefore, the role of MIT-like ACTXs in the venom of funnel-web spiders remains unknown.

\section{Acknowledgements}

This research was partially funded by a UTS Early Career Researcher Grant to SW and an Australian Research Council Discovery Grant to GMN and WCH. The Schering-Plough Research Institute is entirely funded by Schering-Plough Corporation. We are grateful to Dr Roger D. Drinkwater and Ms Jodi-Lea Matheson (presently at Xenome Ltd. PO Box 6295, St. Lucia, QLD 4067, Australia) for providing the sequence for ACTX-Hi:OBf7512.

\section{References}

[1] Bendtsen JD, Nielsen H, von Heijne G, Brunak S. Improved prediction of signal peptides: SignalP 3.0. J Mol Biol 2004;340:783-95.

[2] Boisbouvier J, Albrand JP, Blackledge M, Jaquinod M, Schweitz H, Lazdunski M et al. A structural homologue of colipase in black mamba venom revealed by NMR floating disulphide bridge analysis. J Mol Biol 1998;283:205-19.

[3] Bougis PE, Rochat H, Smith LA. Precursors of Androctonus australis scorpion neurotoxins. Structures of precursors, processing outcomes, and expression of a functional recombinant toxin II. J Biol Chem 1989;264:19259-65.

[4] Chen T, Farragher S, Bjourson AJ, Orr DF, Rao P, Shaw C. Granular gland transcriptomes in stimulated amphibian skin secretions. Biochem J 2003;371:125-30.

[5] Cheng MY, Bullock CM, Li C, Lee AG, Bermak JC, Belluzzi J et al. Prokineticin 2 transmits the behavioural circadian rhythm of the suprachiasmatic nucleus. Nature 2002;417:405-10.

[6] Colledge CJ, Hunsperger JP, Imperial JS, Hillyard DR. Precursor structure of omega-conotoxin GVIA determined from a cDNA clone. Toxicon 1992;30:1111-6.

[7] Craig AG, Norberg T, Griffin D, Hoeger C, Akhtar M, Schmidt K et al. Contulakin-G, an O- 
glycosylated invertebrate neurotensin. J Biol Chem 1999;274:13752-9.

[8] Danse JM, Rowan EG, Gasparini S, Ducancel F, Vatanpour H, Young LC et al. On the site by which alpha-dendrotoxin binds to voltage-dependent potassium channels: site-directed mutagenesis reveals that the lysine triplet 28-30 is not essential for binding. FEBS Lett 1994;356:153-8.

[9] Diniz MR, Paine MJ, Diniz CR, Theakston RD, Crampton JM. Sequence of the cDNA coding for the lethal neurotoxin Tx1 from the Brazilian "armed" spider Phoneutria nigriventer predicts the synthesis and processing of a preprotoxin. J Biol Chem 1993;268:15340-2.

[10] Fletcher JI, Chapman BE, Mackay JP, Howden MEH, King GF. The structure of versutoxin $(\delta-$ atracotoxin-Hv1): implications for binding of site-3 toxins to the voltage-gated sodium channel. Structure 1997;5:1525-35.

[11] Fletcher JI, Smith R, O'Donoghue SI, Nilges M, Connor M, Howden ME et al. The structure of a novel insecticidal neurotoxin, $\omega$-atracotoxin-HV1, from the venom of an Australian funnel web spider. Nature Struct Biol 1997;4:559-66.

[12] Fletcher JI, Dingley AJ, Smith R, Connor M, Christie MJ, King GF. High-resolution solution structure of gurmarin, a sweet-taste-suppressing plant polypeptide. Eur $\mathrm{J}$ Biochem 1999;264:525-33.

[13] Glinka A, Wu W, Delius H, Monaghan AP, Blumenstock C, Niehrs C. Dickkopf-1 is a member of a new family of secreted proteins and functions in head induction. Nature 1998;391:357-62.

[14] Heitz A, Le-Nguyen D, Chiche L. Min-21 and min-23, the smallest peptides that fold like a cystine-stabilized beta-sheet motif: design, solution structure, and thermal stability. Biochemistry 1999;38:10615-25.

[15] Hutchinson EG, Thornton JM. PROMOTIF--a program to identify and analyze structural motifs in proteins. Protein Sci 1996;5:212-20.

[16] Joubert FJ, Strydom DJ. Snake venom. The amino acid sequence of protein A from Dendroaspis polylepis polylepis (black mamba) venom. Hoppe Seylers Z Physiol Chem 1980;361:1787-94.

[17] Kalapothakis E, Penaforte CL, Leao RM, Cruz JS, Prado VF, Cordeiro MN et al. Cloning, cDNA sequence analysis and patch clamp studies of a toxin from the venom of the armed spider (Phoneutria nigriventer). Toxicon 1998;36:1971-80.

[18] Kalus W, Zweckstetter M, Renner C, Sanchez Y, Georgescu J, Grol M et al. Structure of the IGF-binding domain of the insulin-like growth factor-binding protein-5 (IGFBP-5): implications for IGF and IGF-I receptor interactions. EMBO J 1998;17:6558-72.

[19] Kaser A, Winklmayr M, Lepperdinger G, Kreil G. The AVIT protein family. EMBO Rep 
2003;4:469-73.

[20] Kreil G, Mollay C, Kaschnitz R, Haiml L, Vilas U. Prepromelittin: specific cleavage of the preand the propeptide in vitro. Ann N Y Acad Sci 1980;343:338-46.

[21] Lai R, Liu H, Lee WH, Zhang Y. Two novel Bv8-like peptides from skin secretions of the toad Bombina maxima. Comp Biochem Physiol B Biochem Mol Biol 2003;134:509-14.

[22] LeCouter J, Kowalski J, Foster J, Hass P, Zhang Z, Dillard-Telm L et al. Identification of an angiogenic mitogen selective for endocrine gland endothelium. Nature 2001;412:877-84.

[23] LeCouter J, Ferrara N. EG-VEGF and the concept of tissue-specific angiogenic growth factors. Semin Cell Dev Biol 2002;13:3-8.

[24] LeCouter J, Lin R, Ferrara N. The role of EG-VEGF in the regulation of angiogenesis in endocrine glands. Cold Spring Harb Symp Quant Biol 2002;67:217-21.

[25] LeCouter J, Lin R, Ferrara N. Endocrine gland-derived VEGF and the emerging hypothesis of organ-specific regulation of angiogenesis. Nat Med 2002;8:913-7.

[26] LeCouter J, Lin R, Frantz G, Zhang Z, Hillan K, Ferrara N. Mouse endocrine gland-derived vascular endothelial growth factor: a distinct expression pattern from its human ortholog suggests different roles as a regulator of organ-specific angiogenesis. Endocrinology 2003; 144:2606-16.

[27] Li M, Bullock CM, Knauer DJ, Ehlert FJ, Zhou QY. Identification of two prokineticin cDNAs: recombinant proteins potently contract gastrointestinal smooth muscle. Mol Pharmacol 2001;59:692-98.

[28] Lin DC, Bullock CM, Ehlert FJ, Chen JL, Tian H, Zhou QY. Identification and molecular characterization of two closely related G protein-coupled receptors activated by prokineticins/endocrine gland vascular endothelial growth factor. J Biol Chem 2002;277:1927680.

[29] Masuda Y, Takatsu Y, Terao Y, Kumano S, Ishibashi Y, Suenaga M et al. Isolation and identification of EG-VEGF/prokineticins as cognate ligands for two orphan G-protein-coupled receptors. Biochem Biophys Res Commun 2002;293:396-402.

[30] Mattinen ML, Kontteli M, Kerovuo J, Linder M, Annila A, Lindeberg G et al. Three-dimensional structures of three engineered cellulose-binding domains of cellobiohydrolase I from Trichoderma reesei. Protein Sci 1997;6:294-303.

[31] Melchiorri D, Bruno V, Besong G, Ngomba RT, Cuomo L, De Blasi A et al. The mammalian homologue of the novel peptide Bv8 is expressed in the central nervous system and supports neuronal survival by activating the MAP kinase/PI-3-kinase pathways. Eur J Neurosci 
2001;13:1694-702.

[32] Mollay C, Wechselberger C, Mignogna G, Negri L, Melchiorri P, Barra D et al. Bv8, a small protein from frog skin and its homologue from snake venom induce hyperalgesia in rats. Eur $\mathrm{J}$ Pharmacol 1999;374:189-96.

[33] Morris AL, MacArthur MW, Hutchinson EG, Thornton JM. Stereochemical quality of protein structure coordinates. Proteins 1992;12:345-64.

[34] Negri L, Lattanzi R, Giannini E, Metere A, Colucci M, Barra D et al. Nociceptive sensitization by the secretory protein Bv8. Brit J Pharmacol 2002;137:1147-54.

[35] Nicholson GM, Little M, Birinyi-Strachan LC. Structure and function of $\delta$-atracotoxins: lethal neurotoxins targeting the voltage-gated sodium channel. Toxicon 2004;43:587-99.

[36] Omecinsky DO, Holub KE, Adams ME, Reily MD. Three-dimensional structure analysis of $\mu$ agatoxins: further evidence for common motifs among neurotoxins with diverse ion channel specificities. Biochemistry 1996;35:2836-44.

[37] Pescatori M, Bradbury A, Bouet F, Gargano N, Mastrogiacomo A, Grasso A. The cloning of a cDNA encoding a protein (latrodectin) which co-purifies with the alpha-latrotoxin from the black widow spider Latrodectus tredecimguttatus (Theridiidae). Eur J Biochem 1995;230:322-8.

[38] Schwartz TW. The processing of peptide precursors. 'Proline-directed arginyl cleavage' and other monobasic processing mechanisms. FEBS Lett 1986;200:1-10.

[39] Schweitz H, Pacaud P, Diochot S, Moinier D, Lazdunski M. MIT 1 , a black mamba toxin with a new and highly potent activity on intestinal contraction. FEBS Lett 1999;461:183-8.

[40] Shu Q, Lu SY, Gu XC, Liang SP. The structure of spider toxin huwentoxin-II with unique disulfide linkage: evidence for structural evolution. Protein Sci 2002;11:245-52.

[41] Sollod BL, Wilson D, Zhaxybayeva O, Gogarten JP, Drinkwater R, King GF. Were arachnids the first to use combinatorial peptide libraries? Peptides 2005;26:131-9.

[42] Szeto TH, Wang X-H, Smith R, Connor M, Christie MJ, Nicholson GM et al. Isolation of a funnel web spider polypeptide with homology to mamba intestinal toxin 1 and the embryonic head inducer Dickkopf1. Toxicon 2000;38:429-42.

[43] Tedford HW, Maggio F, Sollod BL, King GF. Australian funnel-web spiders: master insecticide chemists. Toxicon 2004;43:601-18.

[44] Tytgat J, Chandy KG, Garcia ML, Gutman GA, Martin-Eauclaire MF, van der Walt JJ et al. A unified nomenclature for short-chain peptides isolated from scorpion venoms: alpha-KTx molecular subfamilies. Trends Pharmacol Sci 1999;20:444-7.

[45] van Tilbeurgh H, Sarda L, Verger R, Cambillau C. Structure of the pancreatic lipase-procolipase 
complex. Nature 1992;359:159-62.

[46] Wang DY, Kumar S, Hedges SB. Divergence time estimates for the early history of animal phyla and the origin of plants, animals and fungi. Proc R Soc Lond B Biol Sci 1999;266:163-71.

[47] Wang X-H, Connor M, Smith R, Maciejewski MW, Howden MEH, Nicholson GM et al. Discovery and characterization of a family of insecticidal neurotoxins with a rare vicinal disulfide bridge. Nature Struct Biol 2000;7:505-13.

[48] Wechselberger C, Puglisi R, Engel E, Lepperdinger G, Boitani C, Kreil G. The mammalian homologues of frog Bv8 are mainly expressed in spermatocytes. FEBS Lett 1999;462:177-81.

\section{Figure Legends}

Fig. 1. RP-HPLC chromatograms of MIT-like ACTX spider peptides. RP-HPLC chromatograms of whole venoms from (A) a single juvenile H. sp. 20 ("Illawarra") (B) an adult female H. infensa: Orchid Beach and (C) pooled adult male and female H. versuta, indicating MIT-like ACTX components.

Fig. 2. Nucleotide and corresponding amino acid sequences of MIT-like ACTX. (A) Nucleotide identities are boxed in black, with the start, peptide N-terminus and stop codons boxed in grey. (B) Amino acid identities boxed in grey with conservative substitutions in grey italic text. (C) Schematic representation of the leader peptide, mature peptide and C-terminal tail proposed to be post-translationally cleaved (shaded box).

Fig. 3. Sequence alignment of MIT-like ACTX spider peptides with the AVIT protein family. Sequence identities relative to ACTX-Hvf17 are shown in grey boxes, while conservative substitutions are in grey italic text. Lines connecting pairs of Cys residues above the sequences indicate the disulfide bonds determined for MIT1. Abbreviations: PRTx16C0 and PRTx16C1, non-toxic peptides from the spider P. reidyi (SWISS-PROT data bank accession numbers P83893 and P83997); Bv8 and Bm8, skin secretion proteins from the toad Bombina variegata and B. maxima, respectively [4, 21, 32]; MIT1 [16, 39]; PK1 and PK2, prokineticin 1 and 2, respectively. Prefixes: r, rat [29]; m, mouse; and h, human [22, 27, 48]; b, bovine (GenBank accession number AY192558) and f, fugu (see [19]). All mammalian and piscine prokineticin sequences are deduced from cloned cDNAs. The lower right table shows the peptide length as well as \% identity and homology (including conserved substitutions) relative to ACTX-Hvf17.

Fig. 4. Effects of ACTX-Hvf17 on smooth muscle contractility. (A-C) Typical smooth muscle 
tension traces showing lack of effect of ACTX-Hvf17 on (Aa) field stimulated guinea-pig ileum longitudinal muscle, (Ab) hPK1 responses in guinea-pig ileum longitudinal muscle, (B) rat stomach fundus longitudinal muscle, and (C) rat aortic strips. Markers beneath each trace indicate the bath application of either $1 \mu \mathrm{M}$ ACh, $4 \mathrm{nM}$ hPK1, or $50 \mathrm{mM} \mathrm{KCl}$ (grey arrowheads), $1 \mu \mathrm{M}$ ACTX-Hvf17 (black arrowheads) or washout with Krebs solution (white arrowheads). (Ac) Responses of guinea-pig ileum longitudinal muscle to $4 \mathrm{nM}$ hPK1 expressed as a percentage of $1 \mu \mathrm{M}$ ACh before (open column) and following incubation with $1 \mu \mathrm{M}$ ACTX-Hvf17 (grey column). Data represent the mean $\pm \mathrm{SE}$ of three individual experiments.

Fig. 5. Pharmacological characterization of ACTX-Hvf17 on hPKR1 receptors expressed in HEK293 cells. (A) Panel shows dose-dependent increases in $\left[\mathrm{Ca}^{2+}\right]_{i}$ produced by hPK1 (closed circles) while ACTX-Hvf17 (open circles) failed to alter resting calcium flux at concentrations up to $100 \mathrm{nM}$. (B) Antagonist activity assay of ACTX-Hvf17 with hPK1. Increasing concentrations of ACTX-Hvf17 were incubated with a fixed concentration of hPK1 $(10 \mathrm{nM})$. Data are means $\pm \mathrm{SE}$ of $4-5$ individual experiments.

Fig. 6. Modeling of MIT-like ACTX and AVIT family peptides. (A) Schematic view of the NMR structure of MIT1 (PDB file 1IMT) showing the location of $\beta$-strands (cyan arrows) and N-terminal $\alpha$ helix (green/yellow). The N- and C- termini are labeled and disulfides bridges are shown as red tubes. (B) Superimposed 3D structure of MIT1 (green) and putative structure of ACTX-Hvf17 (yellow). Loop 3 from MIT1 [CGKGTCC] and from ACTX-Hvf17 [CGAGHCC] superimpose with an rmsd over the backbone atoms of 0.229. (C-D) Surface charge representations of six modeled MIT-like ACTX and AVIT family peptides as labeled compared with the NMR structure of MIT1 below. The colored regions on the surfaces represent positively charged (red), negatively charged (blue) and hydrophobic regions (white). Panel D shows models rotated $180^{\circ}$ around the $y$-axis with comparison to models in panel $\mathrm{C}$. The AVIT family peptides Bv8, rPK1, rPK2 and MIT1 all show a similar surface charge pattern to each other whereas the MIT-like ACTX family peptides have very different surface charge patterns to the AVIT family peptides as well as themselves.

Fig. 7. The ancestral disulfide-directed $\beta$-hairpin fold. Schematic representations of (Aa) the ancient DDH fold, and (Ab) the more elaborate ICK fold, showing $\beta$-sheets (arrows), disulfide bonds (horizontal lines) and position of loops numbered from the N-terminus (' $N$ ') to the C-terminus (' $C$ '). (B) 
Comparison of MIT-like ACTX with other selected eukaryotic disulfide-directed $\beta$-hairpin (DDH) domains. Domains are grouped into those that occur in isolated (I), duplicated (D) and/or fused (F) form as indicated on the left of the panel. The names of AVIT family proteins are highlighted in grey. Abbreviations: $\kappa-A C T X-H v 1 c, \kappa-a t r a c o t o x i n-H v 1 c$ from the venom of the funnel-web spider $H$. versuta [47]; $\mu$-Aga I, $\mu$-agatoxin I from the venom of the unrelated American funnel-web spider Agelenopsis aperta [36]; HWTX-II, huwentoxin-II from the venom of the Chinese bird-eating spider Ornithoctonus huwena [40]; gurmarin, from the plant Gymnema sylvestre [12]; mBv8, mouse Bv8 [48]; hBv8, human Bv8 [48]; PCL, porcine colipase [45]; hDkk-1, human Dickkopf-1 embryonic head inducer (residues 183-266) [13]; IGFBP-5, insulin-like growth factor binding protein 5 [18]; CBD-CBHI, cellulose binding domain of cellobiohydrolase I from the fungus Trichoderma reesei [30]. Remaining abbreviations are the same as for Fig. 3. The structures of all these domains have been determined except the MIT-like ACTX, PRTx16C0, PRTx16C1, Bv8, Bm8a-f, prokineticins and hDkk-1, whose inclusion in this figure is speculative. Note the strong conservation of residues in loop 3 between MITlike ACTX family and AVIT family proteins with a consensus sequence of CGXGXCC. 'N-term' and 'C-term' indicate the N-terminal and C-terminal DDH folds in proteins where the domain has been duplicated. 
Fig 1
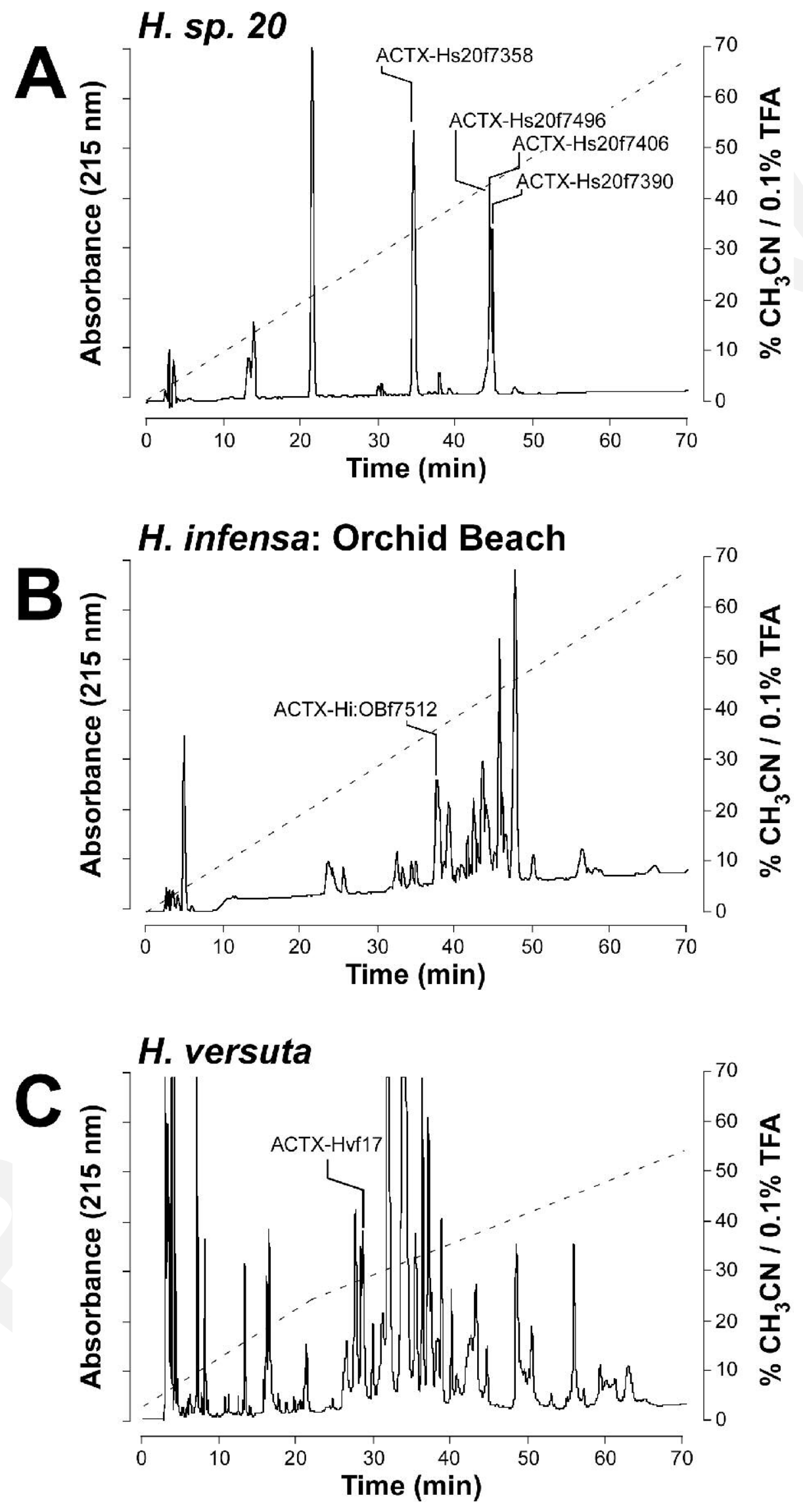
Fig. 2

\section{A}

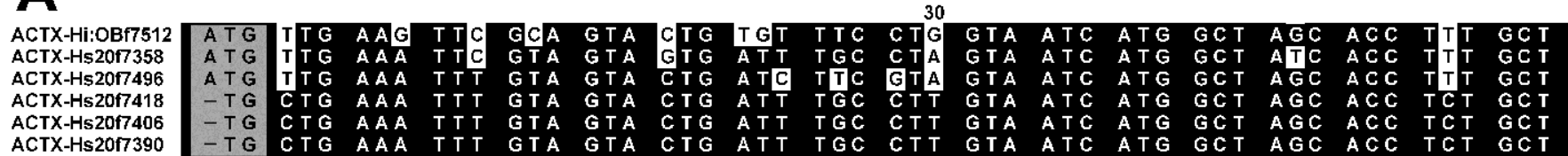

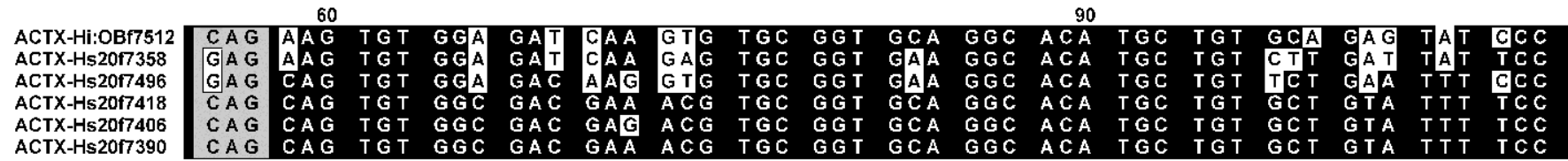

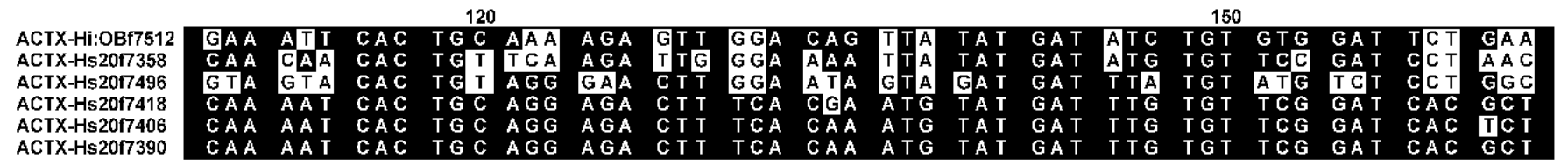

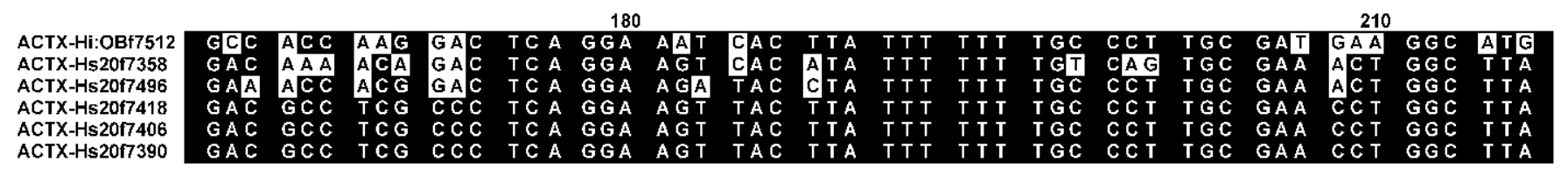

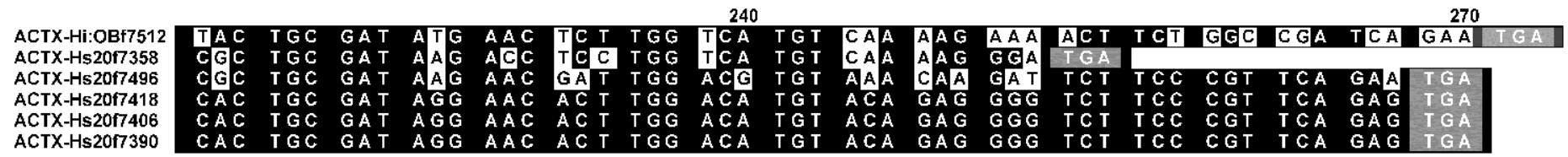

B

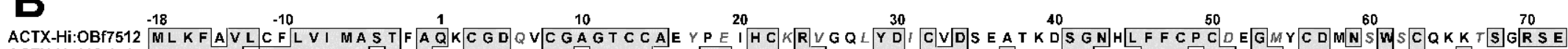

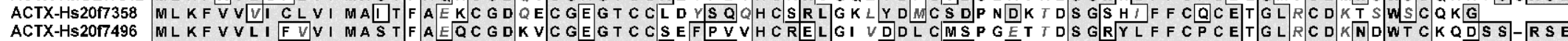

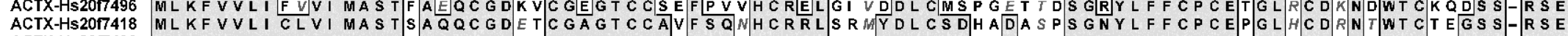

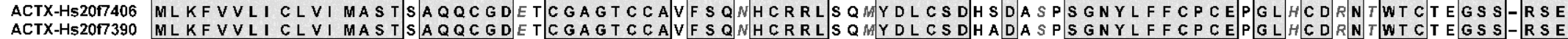

C.

Signal Peptide (18 residues)

Mature Peptide (66-68 residues) 
Fig. 3

ACTX-Hvf17

ACTX-Hi:OBf7512

ACTX-Hs20f7496

ACTX-Hs20f7358

ACTX-Hs20f7390

ACTX-Hs20f7406

PRTx16C0

PRT $\times 16 \mathrm{C}$

Bv8

Bm8a

Bm8b

Bm8c

Bm8d

Bm8e

Bm8f

IPK2

mPK2

bPK2

fPK2

FPK1

rPK1
mPK1
hPK1

ACTX-Hvf17

ACTX-Hi:OBf7512

ACTX-Hs20f7496

ACTX-Hs20f7358

ACTX-Hs20f7418

PRT×1600

PRTx16C0
PRTx16C1

Bv8

Bm8a
Bm8b
Bm8c

Bm8c

Bm8d

Bm8

MIT1

rPK2

MPK2

HPK2

bPK2

fPK2

PPK1

mPK1

mPK1

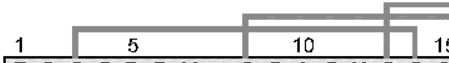

作

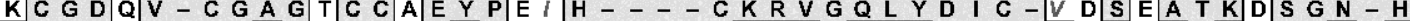
EQ C G DKV - C GE E T C CSEF P V V H - - - CRELG IVDD L C - MS P G ETT T D S GR - Y

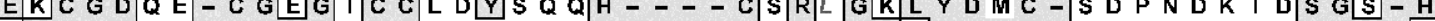

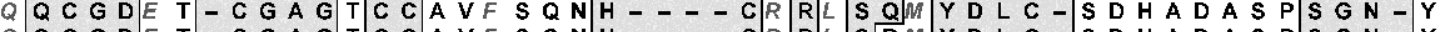
Q Q C G D E T - C G A G T C C C A V F S Q N H - - - - C R R $R$ S S RM Y D L C - S D H A D A S P S G N - Y

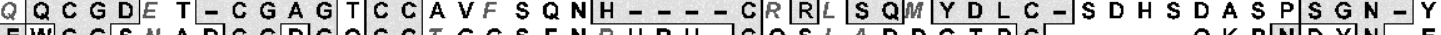
QW I P G Q S C C T N A D L G A V I T G A C D K D D Q C G S G T C C A A S A W S R N I R F C I P L G N S GE D C H P A S H K V P Y D G

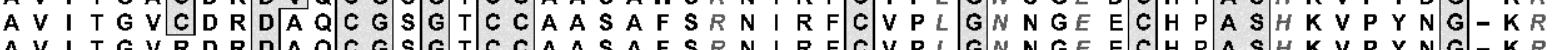
$A V T$ V A V I T V C D R A OCGSGTCCAASAFSR N R F C V P G N NGE EC H P A S H K V P Y NG-KR

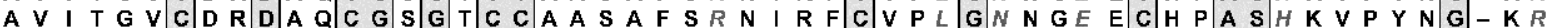
A V I T G V C D R D A Q C C G S G T C C A A S A F S R N I R F C V P L G N N GE E C H P A S H K V P S D G A V I T G A C E R D L Q C G K G TCC CAVS L WIK S V R V C T P V G T S GE D C H P A S H K I P F S G Q RK A V I T G A C D K D S OCG G G M C C A V S I WVKS I R I C T PMG Q V G G S C H P L T R K V P F W G A V I T G A C D K K D S S Q C G G G M C C A V S I WVKS I R I C T PMG Q V G D

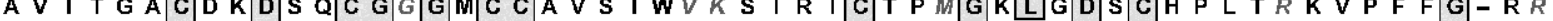

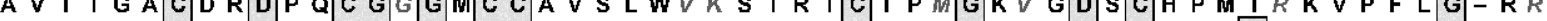

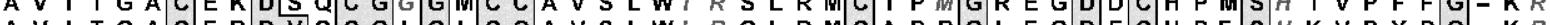
A V T G A E R D Q C G L G L C C A V S LWL R G R M A P R G L E G D E $A V$ T

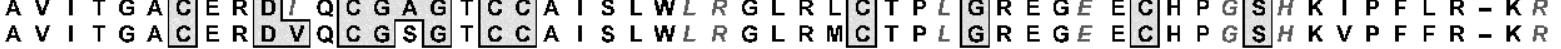

\begin{tabular}{l|r|r|}
\hline 45 & 55 & 60 \\
\hline
\end{tabular}

LFF - C P C D E G M Y C D M N S - - - W S C Q KR TA

F F - C P CDEGM Y C DMN

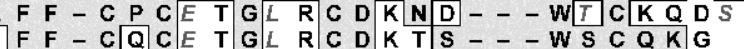

F F - C P C E G H C D R NT- - W T C C T E GS S

L F F - C P C E P G $L$ H C D R

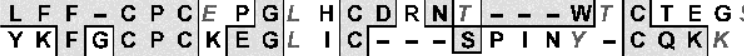

Y K F G C P C K K E GM I C

L S S L C P C K S G L T C S K - S G E KF K C S

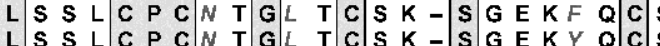

L S S L L C P C $\quad N$ T T G L T T C S K K

L S S L C P C N T G L T C S K - S G E K S Q C S

L S S L C P C N T GL T C P K - S G E KF Q C S

LS S L C P C N T G L T C S K - S G E K Y Q C S

W

H H T C P C L P G L A C L R T S F NRF I C L A R K

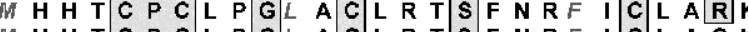

H H

L H H T C P C L P N $L$ S C I P M DE GR A K C L S T Y K Y P D Y Y L

$\begin{array}{llllllll} & \end{array}$

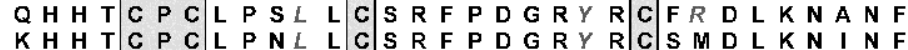

\begin{tabular}{ccc}
\hline Residues & \% Identity & \% Homology \\
\hline 68 & 100 & $\mathbf{1 0 0}$ \\
68 & $\mathbf{8 1}$ & 87 \\
68 & $\mathbf{5 3}$ & 66 \\
66 & $\mathbf{5 0}$ & $\mathbf{5 6}$ \\
68 & $\mathbf{5 0}$ & 66 \\
68 & $\mathbf{5 0}$ & 66 \\
68 & $\mathbf{5 0}$ & 66 \\
64 & $\mathbf{3 4}$ & 46 \\
68 & $\mathbf{3 0}$ & $\mathbf{3 8}$ \\
$\mathbf{7 7}$ & 32 & 44 \\
77 & 31 & 44 \\
77 & 31 & 44 \\
77 & 31 & 44 \\
77 & 31 & 43 \\
77 & 31 & 44 \\
77 & 31 & 44 \\
80 & 31 & 47 \\
81 & $\mathbf{3 1}$ & 46 \\
$\mathbf{8 1}$ & 31 & 46 \\
81 & 29 & 43 \\
81 & $\mathbf{2 8}$ & 44 \\
88 & $\mathbf{2 8}$ & 38 \\
$\mathbf{9 4}$ & $\mathbf{2 8}$ & 38 \\
86 & $\mathbf{2 5}$ & $\mathbf{3 8}$ \\
86 & $\mathbf{2 5}$ & 41 \\
$\mathbf{8 6}$ & $\mathbf{2 5}$ & $\mathbf{3 8}$ \\
\hline & &
\end{tabular}


Fig. 4
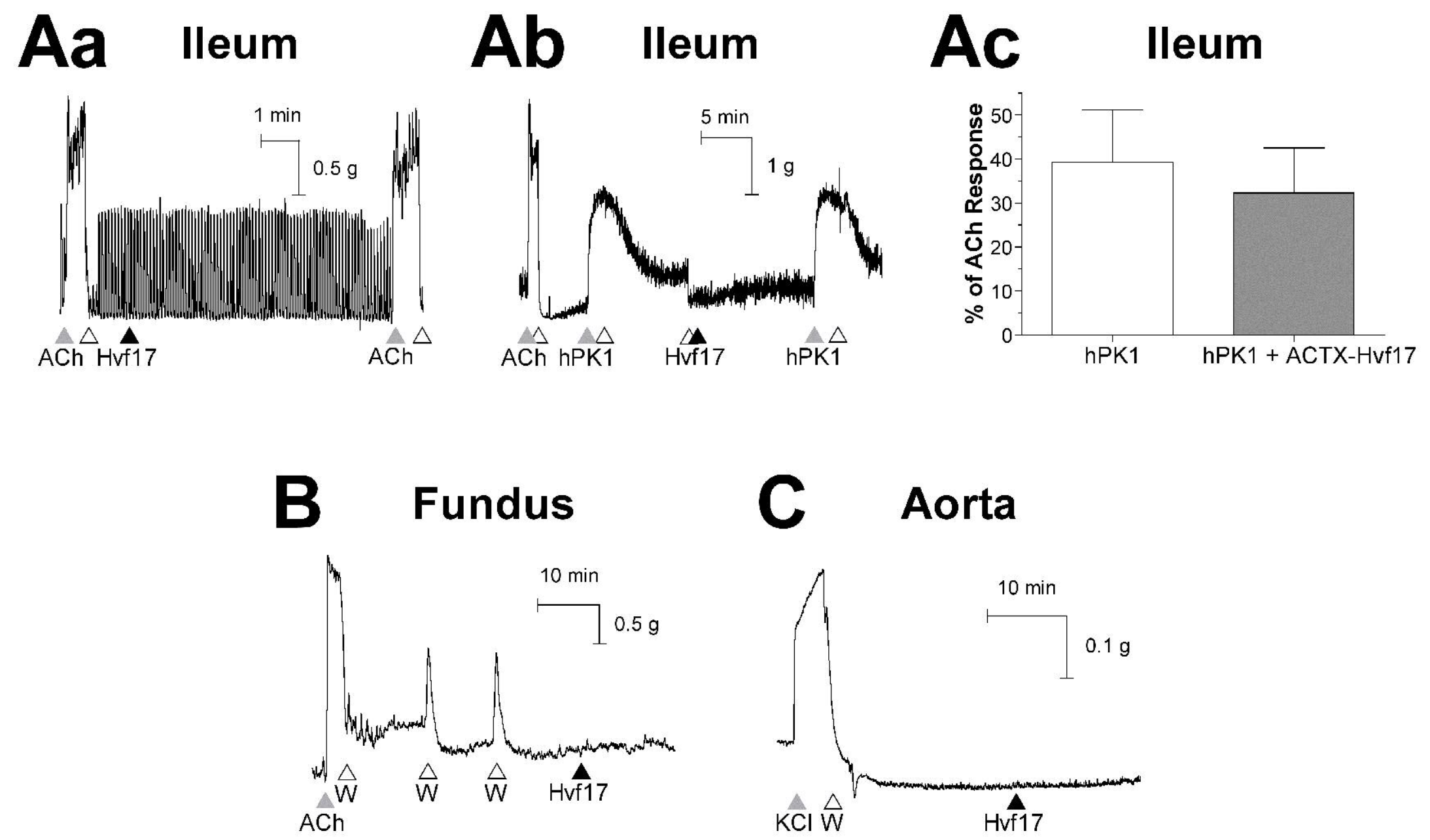
Fig. 5
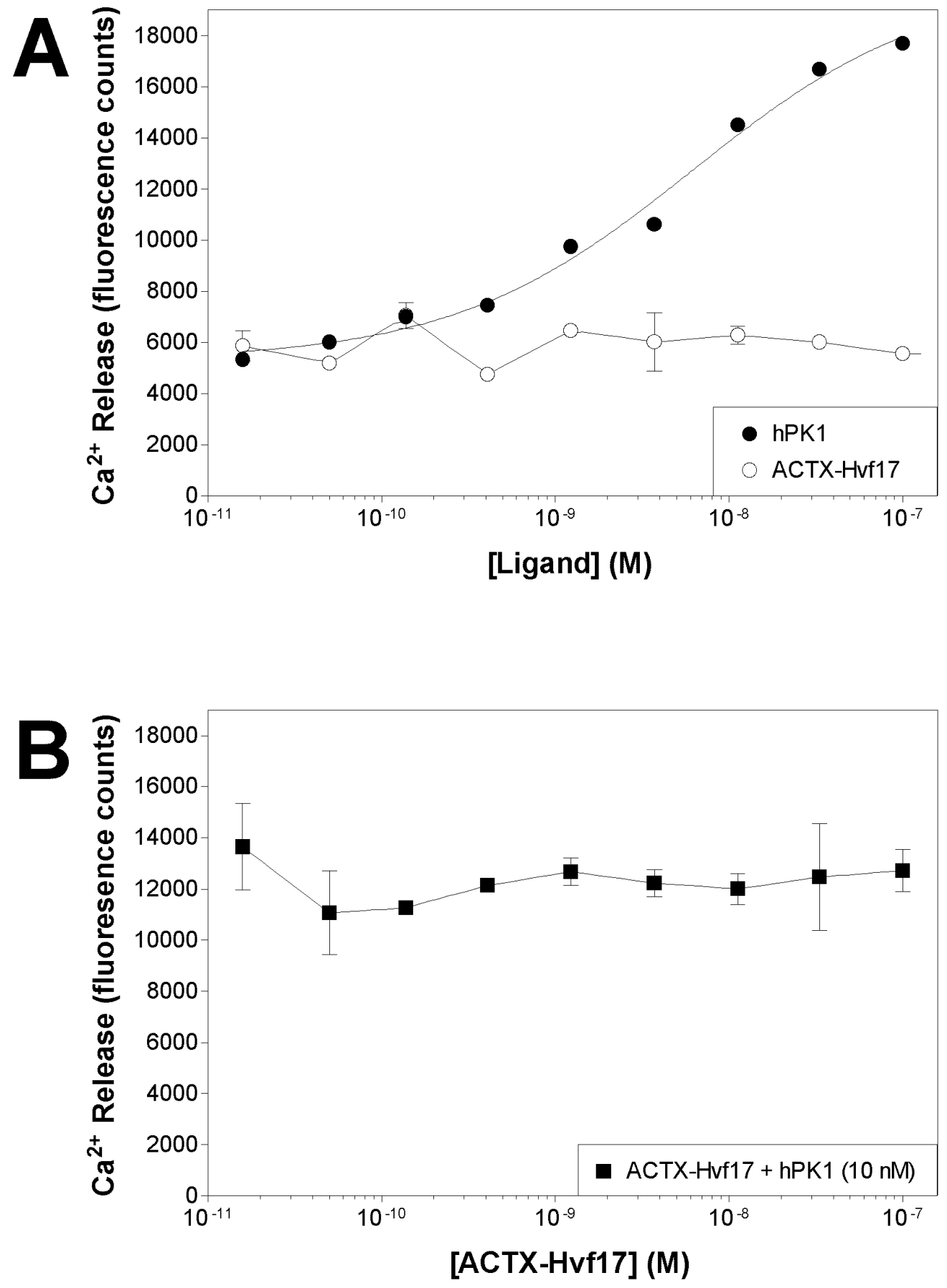
A

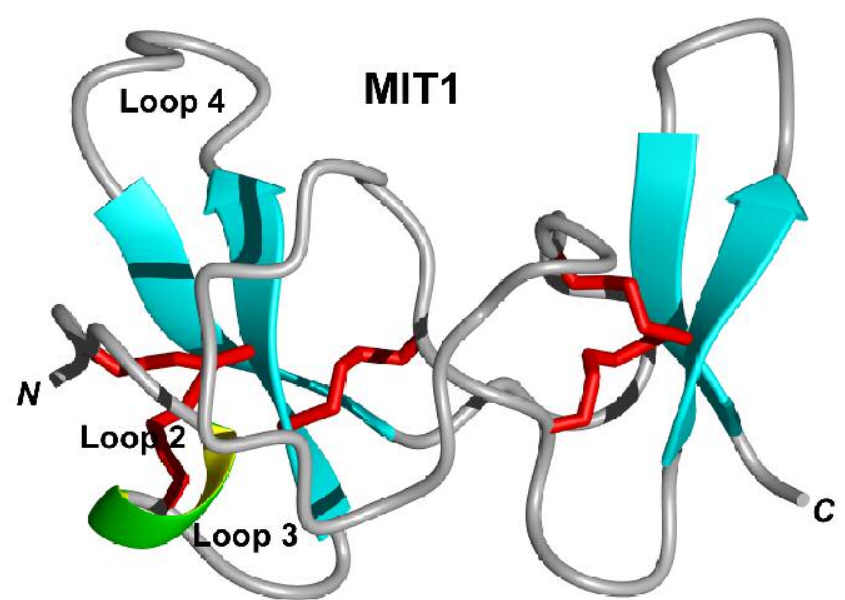

C

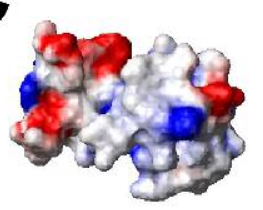
ACTX-Hvf17

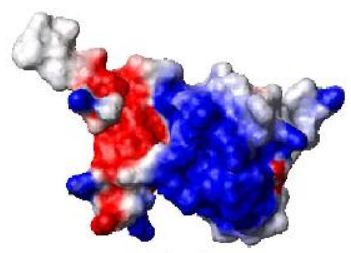

Bv8

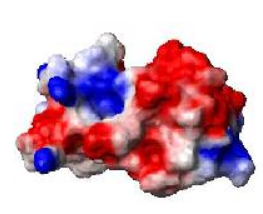

ACTX-Hi:OBf7512
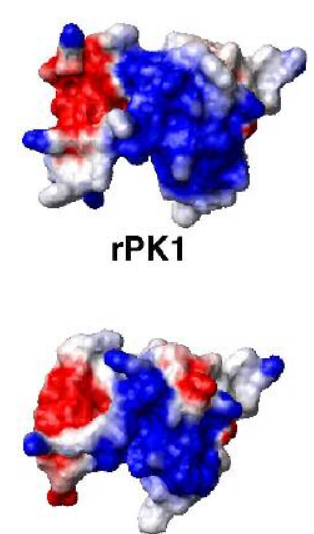

MIT1
B

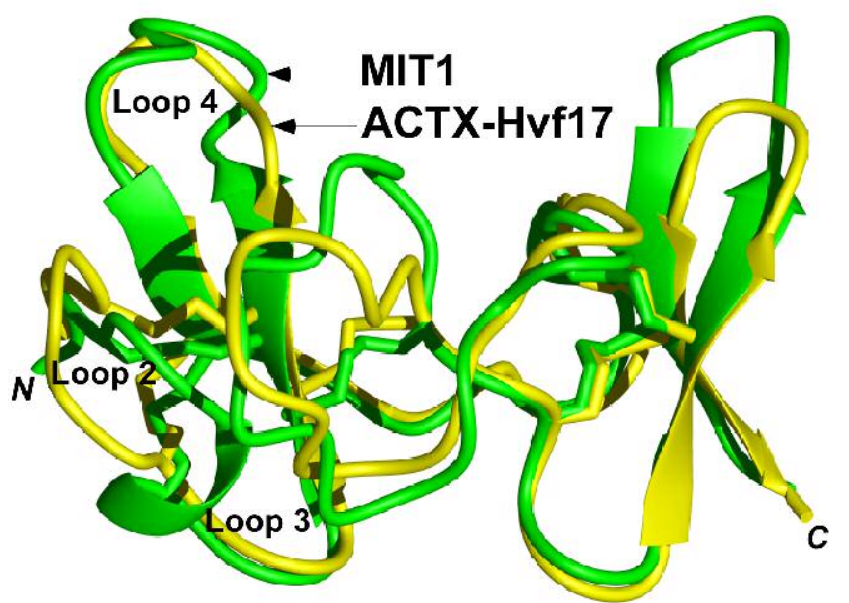

D
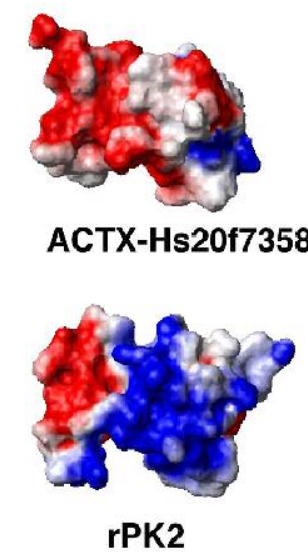

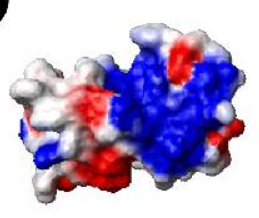

ACTX-Hvf17

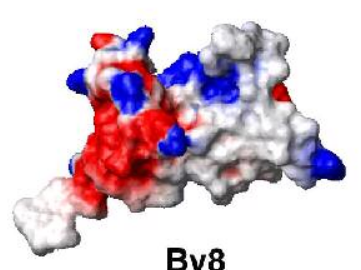

Bv8

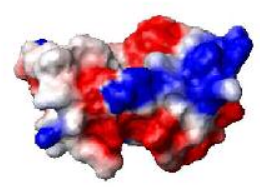

ACTX-Hi:OBf7512

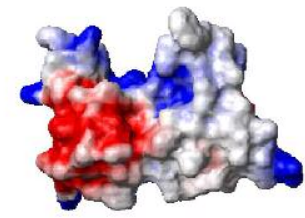

rPK1

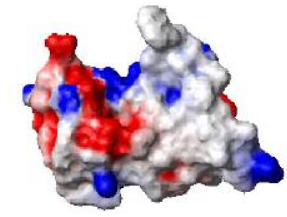

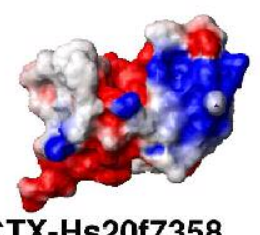

ACTX-Hs20f7358

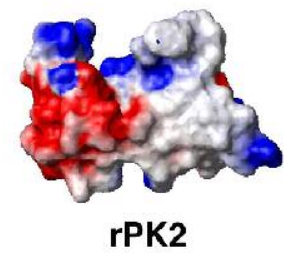

MIT1 


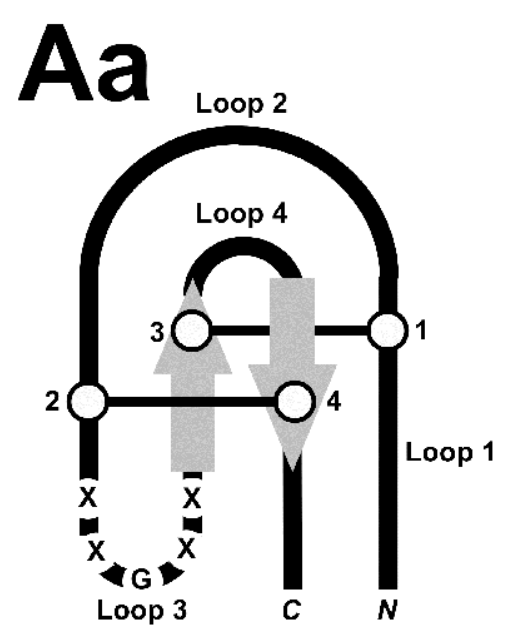

$\mathrm{Ab}$

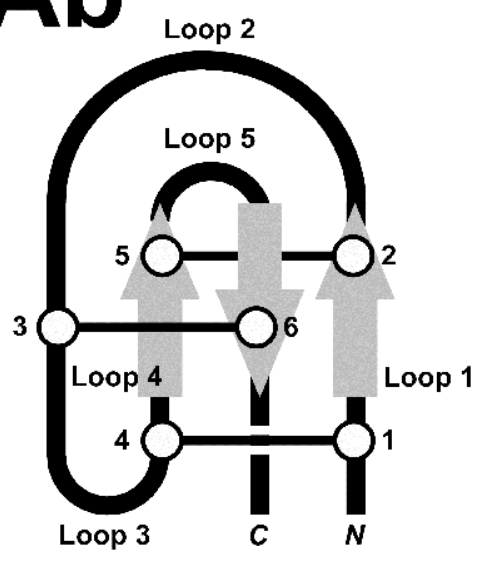

B

ACTX-Hvf1

ACTX-Hi:OBf7512

ACTX-Hs20f7496

ACTX-Hs $20 f 7358$

ACTX-Hs20f7390

ACTX-Hs20f7418

ACTX-Hs20f7406

ACTX-Hs20
PRTX16C0

PRTX16C0
PRTx16C1

K-ACTX-Hv1c

$\mu$-Aga I

HWTX-II

Gurmarin

Bv8

$\mathrm{mBv} 8$
$\mathrm{hBv} 8$

hBv8

Bm8a

$\mathrm{Bm} 8 \mathrm{~b}$

Bm8d

Bm8e

Bm8

rPK2

MPK2

hPK2

bPK2

fPK2

fPK1

rPK1

MPK1

hPK1

MIT1 (N-term)

MIT1 (C-term)

D

PCL (C-term)

hDkk-1 (N-term 1)

hDkk-1 (N-term 2)

CBD-CBH

CONSENSUS
Disulpide 1

Disulphide 2

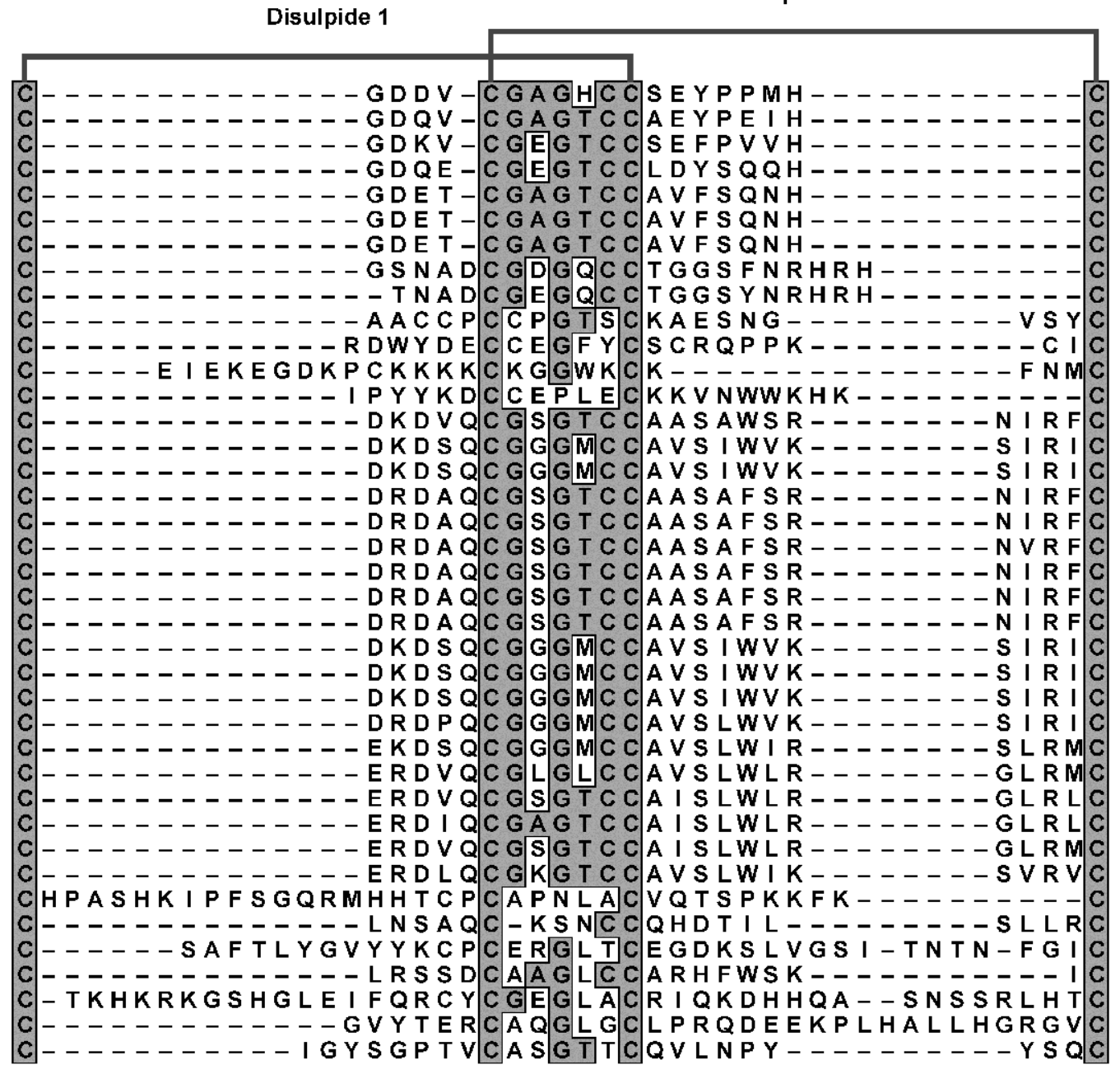

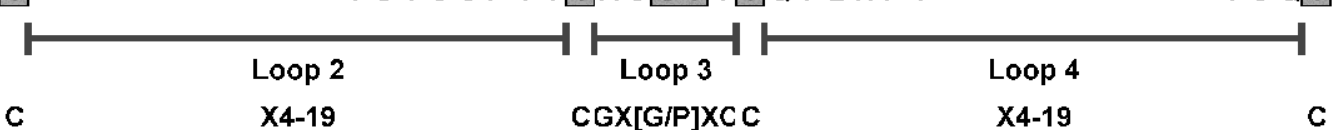

